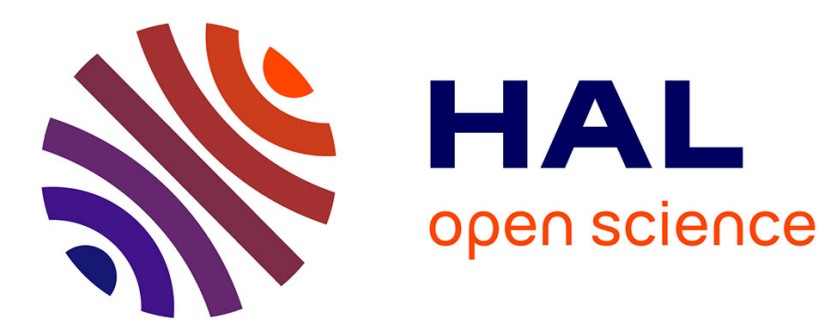

\title{
The Obstacle version of the Geometric Dynamic Programming Principle: Application to the pricing of American options under constraints
}

Bruno Bouchard, Than Nam Vu

\section{- To cite this version:}

Bruno Bouchard, Than Nam Vu. The Obstacle version of the Geometric Dynamic Programming Principle: Application to the pricing of American options under constraints. Applied Mathematics and Optimization, 2010, 61 (2), pp.235-265. 10.1007/s00245-009-9084-y . hal-00343816

\author{
HAL Id: hal-00343816 \\ https://hal.science/hal-00343816
}

Submitted on 2 Dec 2008

HAL is a multi-disciplinary open access archive for the deposit and dissemination of scientific research documents, whether they are published or not. The documents may come from teaching and research institutions in France or abroad, or from public or private research centers.
L'archive ouverte pluridisciplinaire HAL, est destinée au dépôt et à la diffusion de documents scientifiques de niveau recherche, publiés ou non, émanant des établissements d'enseignement et de recherche français ou étrangers, des laboratoires publics ou privés. 


\title{
The American version of the Geometric Dynamic Programming Principle: Application to the pricing of American options under constraints
}

\author{
Bruno Bouchard \\ CEREMADE, Université Paris Dauphine \\ and CREST-ENSAE \\ bouchard@ceremade.dauphine.fr
}

\author{
Vu Thanh Nam \\ CEREMADE, Université Paris Dauphine \\ thanh@ceremade.dauphine.fr
}

December 2, 2008

\begin{abstract}
We provide an American version of the Geometric Dynamic Programming Principle of Soner and Touzi [22] for stochastic target problems. This opens the doors to a wide range of applications, particularly in risk control in finance and insurance, in which a controlled stochastic process has to be maintained in a given set on a time interval $[0, T]$. As an example of application, we show how it can be used to provide a viscosity characterization of the super-heging price of American options under portfolio constraints, without appealing to the standard dual formulation from mathematical finance. In particular, we allow for a degenerate volatility, a case which does not seem to have been studied so far in this context.
\end{abstract}

Key words: Stochastic target problem, discontinuous viscosity solutions, American options.

AMS 1991 subject classifications: Primary 49L25, 60J60; secondary 49L20, 35K55.

\section{Introduction}

A stochastic target problem can be described as follows. Given a set of controls $\mathcal{A}$, a family of $d$ dimensional controlled processes $Z_{0, z}^{\nu}$ with initial condition $Z_{0, z}^{\nu}(0)=z$ and a Borel subset $G$ of $\mathbb{R}^{d}$, find the set $V(0)$ of initial conditions $z$ for $Z_{0, z}^{\nu}$ such that $Z_{0, z}^{\nu}(T) \in G \mathbb{P}$-a.s. for some $\nu \in \mathcal{A}$.

Such a problem appears naturally in mathematical finance. In such applications, $Z_{0, x, y}^{\nu}=\left(X_{0, x}^{\nu}, Y_{0, x, y}^{\nu}\right)$ typically takes values in $\mathbb{R}^{d-1} \times \mathbb{R}, Y_{0, x, y}^{\nu}$ stands for the wealth process, $\nu$ corresponds to the financial strategy, $X_{0, x}^{\nu}$ stands for the price process of some underlying financial assets, and $G$ is the epigraph $\mathcal{E}(g)$ of some Borel map $g: \mathbb{R}^{d-1} \rightarrow \mathbb{R}$. In this case, $V(0)=\left\{(x, y) \in \mathbb{R}^{d-1} \times \mathbb{R}: \exists \nu \in \mathcal{A}\right.$ s.t. $Y_{0, x, y}^{\nu}(T) \geq$ $g\left(X_{0, x}^{\nu}(T)\right) \mathbb{P}$-a.s. $\}$, which, for fixed values of $x$, corresponds to the set of initial endowments from which the European claim of payoff $g\left(X_{0, x}^{\nu}(T)\right)$ paid at time $T$ can be super-replicated.

In the mathematical finance literature, this kind of problems has usually been treated via the so-called dual formulation approach, which allows to reduce this non-standard control problem into a stochastic control problem in standard form, see e.g. [15] and [17]. 
However, such a dual formulation is not always available. This is the case when the dynamics of the underlying financial assets $X^{\nu}$ depends on the control $\nu$ in a non-trivial way. This is also the case for certain kind of models with portfolio constraints, see e.g. [20] for Gamma constraints. Moreover, it applies only in mathematical finance where the duality between super-hedgeable claims and martingale measures can be used, see [15]. In particular, it does not apply in most problems coming from the insurance literature, see e.g. [4] for an example.

In [21] and [22], the authors propose to appeal to a new dynamic programming principle, called geometric, which is directly written on the stochastic target problem:

$$
V(t)=\left\{z \in \mathbb{R}^{d}: \exists \nu \in \mathcal{A} \text { s.t. } Z_{t, z}^{\nu}(\theta) \in V(\theta) \mathbb{P}-\text { a.s. }\right\} \quad \text { for all } \theta \in \mathcal{T}_{[t, T]},
$$

where $V(\theta)$ extends the definition of $V(0)$ to the non trivial time origin $\theta$ and $\mathcal{T}_{[t, T]}$ denotes the set of stopping times taking values in $[t, T]$.

Since then, this principle has been widely used in finance and insurance to provide PDE characterizations to super-hedging type problems, see also [23] for an application to mean curvature flows. Recent results of [7] also allowed to extend this approach to the case where the condition $\mathbb{P}\left[Z_{0, z}^{\nu}(T) \in G\right]=1$ is replaced by the weaker one $\mathbb{P}\left[Z_{0, z}^{\nu}(T) \in G\right] \geq p$, for some fixed $p \in[0,1]$. Similar technologies are used in [6] to study optimal control problems under stochastic target or moment constraints.

Surprisingly, it seems that no American version of this geometric dynamic programming principle has been studied so far. By American version, we mean the following problem: find the set $V(0)$ of initial conditions $z$ for $Z_{0, z}^{\nu}$ such that $Z_{0, z}^{\nu}(t) \in G(t) \forall t \in[0, T] \mathbb{P}$-a.s. for some $\nu \in \mathcal{A}$. Here, $t \mapsto G(t)$ is now a set valued map taking values in the collection of Borel subsets of $\mathbb{R}^{d}$.

The main aim of this paper is to extend the geometric dynamic programming principle to this setting. We shall show in Section 2 that the counterpart of (1.1) for American targets is given by:

$$
V(t)=\left\{z \in \mathbb{R}^{d}: \exists \nu \in \mathcal{A} \text { s.t } Z_{t, z}^{\nu}(\tau \wedge \theta) \in G \stackrel{\tau, \theta}{\bigoplus} V \mathbb{P}-\text { a.s } \forall \tau \in \mathcal{T}_{[t, T]}\right\} \quad \text { for all } \theta \in \mathcal{T}_{[t, T]},
$$

where

$$
G \bigoplus^{\tau, \theta} V:=G(\tau) 1_{\tau \leq \theta}+V(\theta) 1_{\tau>\theta}
$$

In Section 3, we provide an example of application in mathematical finance. Namely, we study the problem of super-hedging an American option under portfolio constraints. The problem of superhedging financial options under constraints has been widely studied since the seminal works of Cvitanic̀ and Karatzas [10] and Broadie et al. [8]. From a practical point of view, it is motivated by two important considerations: 1 . constraints may be imposed by a regulator, 2 . imposing constraints avoids the well-know phenomenon of explosion of the hedging strategy near the maturity when the payoff is discontinuous, see e.g. [19].

Recently, this problem has been further studied by [5] who considered the problem of super-hedging under constraints for barrier type options in a general Markovian setting, thus extending previous results of [19]. A similar analysis has been carried out by [13] for American options and by [14] for perpetual American barrier options both within a Black and Scholes type financial model.

Our analysis can be compared to [24] who considered European type options, and the proofs are actually very close once the dynamic programming (1.1) is replaced by (1.2). The main difference here comes from the fact that we do not impose that the volatility matrix is invertible, an assumption which was also crucial in [5], [8], [10], [13], [14] and [24]. This introduces new technical difficulties which we tackle 
in this particular context but could be transported without major difficulties to the cases discussed in the above quoted papers.

In order to separate the difficulties, we shall restrict to the case where the controls take values in a given convex compact set. The case of unbounded control sets can be handled by using the technology developed in [7].

We conclude this introduction with some notations that will be used all over this paper.

Notations. From now on, we let $(\Omega, \mathcal{F}, \mathbb{P})$ be a probability space endowed with a filtration $\mathbb{F}:=\left(\mathcal{F}_{t}\right)_{t \leq T}$ for some fixed time horizon $T>0$. It is assumed to satisfy the usual assumptions.

Given $0 \leq s \leq t \leq T$, we denote by $\mathcal{T}_{[s, t]}$ the set of $[s, t]$-valued stopping times. We simply write $\mathcal{T}$ for $\mathcal{T}_{[0, T]}$. For any $\theta \in \mathcal{T}$, we let $L_{d}^{p}(\theta)$ be the set of all $p$-integrable $\mathbb{R}^{d}$-valued random variables which are measurable with respect to $\mathcal{F}_{\theta}$. For ease of notations, we set $L_{d}^{p}:=L_{d}^{p}(T)$. We denote by $\mathcal{S}$ the set of $(\theta, \xi) \in \mathcal{T} \times L_{d}^{2}$ such that $\xi \in L_{d}^{2}(\theta)$. Observe that $S:=[0, T] \times \mathbb{R}^{d} \subset \mathcal{S}$. We denote by $\mathbb{H}_{d}^{0}$ the set of all $\mathbb{R}^{d}$-valued cad-lag progressively measurable processes. Inequalities or inclusion between random variables or random sets have to be taken $\mathbb{P}$-a.s. In case of processes, they should be considered $L e b \times \mathbb{P}$-a.e., where Leb is the Lebesgue measure on $[0, T]$. Any element $x$ of $\mathbb{R}^{d}$ is identified to a column vector whose $i$-th component is denoted by $x^{i}$ and Euclidean norm is $|x|, \operatorname{diag}[x]$ is the diagonal matrix whose $i$-th diagonal element is $x^{i}$. Transposition is denoted by '. Given $x, \rho \in \mathbb{R}^{d}$ we set $x \rho:=\left(x^{i} \rho^{i}\right)_{i \leq d}$, $e^{\rho}:=\left(e^{\rho^{i}}\right)_{i \leq d}$ and $x^{\rho}:=\prod_{i=1}^{d}\left(x^{i}\right)^{\rho^{i}}$, whenever the operations are well defined. We denote by $B_{r}(x)$ the open ball of radius $r>0$ and center $x$. For a set $A \subset \mathbb{R}^{d}$, we note $\operatorname{int}(A)$ its interior and $\partial A$ its boundary. Given a smooth map $\varphi$ on $[0, T] \times \mathbb{R}^{d}$, we denote by $\partial_{t} \varphi$ its partial derivatives with respect to its first variable, and by $D \varphi$ and $D^{2} \varphi$ is partial gradient and Hessian matrix with respect to its second variable. For a locally bounded map $v$ on $[0, T] \times(0, \infty)^{d}$, we set

$$
v_{*}(t, x):=\liminf _{\substack{(s, z) \rightarrow(t, x) \\(s, z) \in[0, T) \times(0, \infty)^{d}}} v(s, z) \text { and } v^{*}(t, x):=\limsup _{\substack{(s, z) \rightarrow(t, x) \\(s, z) \in[0, T) \times(0, \infty)^{d}}} v(s, z)
$$

for $(t, x) \in[0, T] \times(0, \infty)^{d}$.

\section{The American geometric dynamic programming principle}

The American stochastic problem consists in describing the set of initial conditions on $Z^{\nu}$ such that $Z^{\nu}(\tau) \in G(\tau)$ for all stopping time $\tau \leq T$ :

$$
V(t):=\left\{z \in \mathbb{R}^{d}: \exists \nu \in \mathcal{A} \text { s.t } Z_{t, z}^{\nu}(\tau) \in G(\tau) \text { for all } \tau \in \mathcal{T}_{[t, T]}\right\}, t \leq T .
$$

Before to state our main result, we now make precise the definitions of the involved quantities. We also recall the natural assumptions that were used in [22] and introduce a new assumption which will allow us to extend their result to our framework: the right-continuity assumption on the target $\mathbf{G}$.

\subsection{Standing Assumptions}

On the target. The map $G$ is a measurable set-valued map from $[0, T]$ to the set $\mathcal{B}_{\mathbb{R}^{d}}$ of Borel sets of $\mathbb{R}^{d}$. It is assumed to be right-continuous in the following sense:

G. Right-continuity of the target. For all sequence $\left(t_{n}, z_{n}\right)_{n}$ of $[0, T] \times \mathbb{R}^{d}$ such that $\left(t_{n}, z_{n}\right) \rightarrow(t, z)$, we have

$$
t_{n} \geq t_{n+1} \text { and } z_{n} \in G\left(t_{n}\right) \forall n \geq 1 \quad \Longrightarrow \quad z \in G(t) .
$$


On the set admissible controls. Given a Borel subset $U$ of an Euclidean space, we denote by $\mathcal{U}$ the set of all progressively measurable processes $\nu:[0, T] \times \Omega \rightarrow U$.

The set of admissible controls $\mathcal{A}$ is defined as a given Borel subset of $\mathcal{U}$ which satisfies the following two conditions:

A1. Stability under concatenation at stopping times: For all $\nu_{1}, \nu_{2} \in \mathcal{A}$ and $\theta \in \mathcal{T}$,

$$
\nu_{1} 1_{[0, \theta)}+\nu_{2} 1_{[\theta, T]} \in \mathcal{A} .
$$

A2. Stability under measurable selection: For any $\theta \in \mathcal{T}$ and any measurable map $\phi:(\Omega, \mathcal{F}(\theta)) \rightarrow$ $\left(\mathcal{A}, \mathcal{B}_{\mathcal{A}}\right)$, there exists $\nu \in \mathcal{A}$ such that

$$
\nu=\phi \text { on }[\theta, T] \times \Omega, L e b \times \mathbb{P}-\text { a.e. },
$$

where $\mathcal{B}_{\mathcal{A}}$ is the set of all Borel subsets of $\mathcal{A}$.

Remark 2.1 The previous assumptions are natural in optimal control, see e.g. [16], and already appear in Soner and Touzi [22]. The assumption $\mathbf{A 2}$ holds whenever $\mathcal{A}$ is a separable metric space, see Lemma 2.1 in $[22]$.

On the state process. The controlled state process is a map from $\mathcal{S} \times \mathcal{A}$ into a subset $\mathcal{Z}$ of $\mathbb{H}_{d}^{0}$

$$
(\theta, \xi, \nu) \in \mathcal{S} \times \mathcal{A} \mapsto Z_{\theta, \xi}^{\nu} \in \mathcal{Z}
$$

As in [22], except for $\mathbf{Z 2}$ which is stated in a stronger form, the state process is assumed to satisfy the following conditions, for all $(\theta, \xi) \in \mathcal{S}$ and $\nu \in \mathcal{A}$ :

Z1. Initial condition: $Z_{\theta, \xi}^{\nu}=0$ on $[0, \theta)$ and $Z_{\theta, \xi}^{\nu}(\theta)=\xi$.

Z2. Consistency with deterministic initial data: For all $(t, z) \in S$ and bounded Borel function $f$, we have

$$
\mathbb{E}\left[f\left(Z_{\theta, \xi}^{\nu}(\vartheta \vee \theta)\right) \mid(\theta, \xi)=(t, z)\right]=\mathbb{E}\left[f\left(Z_{t, z}^{\nu}(\vartheta \vee t)\right)\right]
$$

Z3. Flow property: For $\tau \in \mathcal{T}$ such that $\theta \leq \tau \mathbb{P}$-a.s.:

$$
Z_{\theta, \xi}^{\nu}=Z_{\tau, \mu}^{\nu} \text { on }[\tau, T] \text {, where } \mu:=Z_{\theta, \xi}^{\nu}(\tau) \text {. }
$$

Z4. Causality: Let $\tau$ be defined as in $\mathbf{Z 3}$ and fix $\nu_{1}, \nu_{2} \in \mathcal{A}$. If $\nu_{1}=\nu_{2}$ on $[\theta, \tau]$, then

$$
Z_{\theta, \xi}^{\nu_{1}}=Z_{\theta, \xi}^{\nu_{2}} \text { on }[\theta, \tau]
$$

Z5. Measurability: For any $u \leq T$, the map

$$
(t, z, \nu) \in S \times \mathcal{A} \mapsto Z_{t, z}^{\nu}(u)
$$

is Borel measurable.

\subsection{The American Geometric Dynamic Programming Principle}

We can now state the main result of this section which extends Theorem 3.1. in Soner and Touzi [22].

Theorem 2.1 Suppose that the assumptions $\mathbf{Z 1}-\mathbf{Z 5}, \mathbf{A} 1-\mathbf{A 2}$ and $\mathbf{G}$ are satisfied. Fix $t \leq T$ and $\theta \in \mathcal{T}_{[t, T]}$. Then

$$
V(t)=\left\{z \in \mathbb{R}^{d}: \exists \nu \in \mathcal{A} \text { s.t. } Z_{t, z}^{\nu}(\tau \wedge \theta) \in G \stackrel{\tau, \theta}{\bigoplus} V \forall \tau \in \mathcal{T}_{[t, T]}\right\},
$$

where

$$
G \bigoplus^{\tau, \theta} V:=G(\tau) 1_{\tau \leq \theta}+V(\theta) 1_{\tau>\theta}
$$


The above result states that not only $Z_{t, z}^{\nu}(\tau) \in G(\tau)$ for all $\tau \in \mathcal{T}_{[t, T]}$ but also that $Z_{t, z}^{\nu}(\theta)$ should belong to $V(\theta)$, the set of initial data $\xi$ such that $\mathcal{G}(\theta, \xi) \neq \emptyset$, where

$$
\mathcal{G}(t, z):=\left\{\nu \in \mathcal{A}: Z_{t, z}^{\nu}(\tau) \in G(\tau) \text { for all } \tau \in \mathcal{T}_{[t, T]}\right\},(t, z) \in S
$$

To conclude this section, let us discuss some particular cases. In the discussions below, we assume that the conditions of Theorem 2.1 hold.

Example 2.1 (One sided constraint) Let $Z_{t, x, y}^{\nu}$ be of the form $\left(X_{t, x, y}^{\nu}, Y_{t, x, y}^{\nu}\right)$ where $X_{t, x, y}^{\nu}$ takes values in $\mathbb{R}^{d-1}$ and $Y_{t, x, y}^{\nu}$ takes values in $\mathbb{R}$. Assume further that:

- $G(t):=\left\{(x, y) \in \mathbb{R}^{d-1} \times \mathbb{R}: y \geq g(t, x)\right)$, for some Borel measurable map $g:[0, T] \times \mathbb{R}^{d-1} \mapsto \mathbb{R}$.

- The sets $\Gamma(t, x):=\{y \in \mathbb{R}:(x, y) \in V(t)\}$ are half spaces, i.e.: $y_{1} \geq y_{2}$ and $y_{2} \in \Gamma(t, x) \Rightarrow y_{1} \in$ $\Gamma(t, x)$, for any $(t, x) \in[0, T] \times \mathbb{R}^{d-1}$.

Note that the last condition is satisfied if $X^{\nu}$ does not depend on the initial condition $y$ and when $y \mapsto Y_{t, x, y}^{\nu}$ is non-decreasing.

In this case, the associated value function

$$
v(t, x):=\inf \Gamma(t, x), \quad(t, x) \in[0, T] \times \mathbb{R}^{d-1}
$$

completely characterizes the (interior of the) set $V(t)$ and a version of Theorem 2.1 can be stated in terms of the value function $v$ :

(DP1) If $y>v(t, x)$, then there exists $\nu \in \mathcal{A}$ such that

$$
Y_{t, x, y}^{\nu}(\theta) \geq v\left(\theta, X_{t, x, y}^{\nu}(\theta)\right) \text { for all } \theta \in \mathcal{T}_{[t, T]} .
$$

(DP2) If $y<v(t, x)$, then $\exists \tau \in \mathcal{T}_{[t, T]}$ such that

$$
\mathbb{P}\left[Y_{t, x, y}^{\nu}(\theta \wedge \tau)>v\left(\theta, X_{t, x, y}^{\nu}(\theta)\right) 1_{\theta<\tau}+g\left(\tau, X_{t, x, y}^{\nu}(\tau)\right) 1_{\theta \geq \tau}\right]<1
$$

for all $\theta \in \mathcal{T}_{[t, T]}$ and $\nu \in \mathcal{A}$.

Note that, by definition of $v$ we have $v \geq g$, which explains why the constraints $Y_{t, x, y}^{\nu}(\tau) \geq g\left(\tau, X_{t, x, y}^{\nu}(\tau)\right)$ needs not to appear in (DP1).

Example 2.2 (Two sided constraint) Let $Z_{t, x, y}^{\nu}$ be as in Example 2.1. Assume that $G$ satisfies:

- $\left.G(t):=\left\{(x, y) \in \mathbb{R}^{d-1} \times \mathbb{R}: \underline{g}(t, x) \leq y \leq \bar{g}(t, x)\right)\right\}$, for some Borel measurable maps $\underline{g}, \bar{g}$ : $[0, T] \times \mathbb{R}^{d-1} \mapsto \mathbb{R}$ satisfying $\underline{g} \leq \bar{g}$ on $[0, T] \times \mathbb{R}^{d-1}$.

- The sets $\Gamma(t, x):=\{y \in \mathbb{R}:(x, y) \in V(t)\}$ are convex, i.e.: $\lambda \in[0,1]$ and $y_{1}, y_{2} \in \Gamma(t, x) \Rightarrow$ $\lambda y_{1}+(1-\lambda) y_{2} \in \Gamma(t, x)$, for any $(t, x) \in[0, T] \times \mathbb{R}^{d-1}$.

In this case, the associated value functions

$$
\underline{v}(t, x):=\inf \Gamma(t, x) \quad \text { and } \quad \bar{v}(t, x):=\sup \Gamma(t, x), \quad(t, x) \in[0, T] \times \mathbb{R}^{d-1}
$$

completely characterize the (interior of the) set $V(t)$ and a version of Theorem 2.1 can be stated in terms of these value functions: 
(DP1) If $y \in(\underline{v}(t, x), \bar{v}(t, x))$, then there exists $\nu \in \mathcal{A}$ such that

$$
\underline{v}\left(\theta, X_{t, x, y}^{\nu}(\theta)\right) \leq Y_{t, x, y}^{\nu}(\theta) \leq \bar{v}\left(\theta, X_{t, x, y}^{\nu}(\theta)\right) \text { for all } \theta \in \mathcal{T}_{[t, T]} .
$$

(DP2) If $y \notin[\underline{v}(t, x), \bar{v}(t, x)]$, then $\exists \tau \in \mathcal{T}_{[t, T]}$ such that

$$
\mathbb{P}\left[Y_{t, x, y}^{\nu}(\theta \wedge \tau) \in(\underline{v}, \bar{v})\left(\theta, X_{t, x, y}^{\nu}(\theta)\right) 1_{\theta<\tau}+(\underline{g}, \bar{g})\left(\tau, X_{t, x, y}^{\nu}(\tau)\right) 1_{\theta \geq \tau}\right]<1,
$$

for all $\theta \in \mathcal{T}_{[t, T]}$ and $\nu \in \mathcal{A}$.

\subsection{Proof of Theorem 2.1}

The proof follows from similar argument as the one used in Soner and Touzi [22], which we adapt to our context.

Given $t \in[0, T]$, we have to show that $V(t)=\bar{V}(t)$ where

$$
\bar{V}(t):=\left\{z \in \mathbb{R}^{d}: \exists \nu \in \mathcal{A} \text { s.t } Z_{t, z}^{\nu}(\tau \wedge \theta) \in G \bigoplus^{\tau, \theta} V \text { for all } \tau \in \mathcal{T}_{[t, T]}\right\},
$$

for some $\theta \in \mathcal{T}_{[t, T]}$.

We split the proof in several Lemmas. From now on, we assume that the conditions of Theorem 2.1 hold.

Lemma 2.1 $V(t) \subset \bar{V}(t)$.

Proof. Fix $z \in V(t)$ and $\nu \in \mathcal{G}(t, z)$, i.e. such that $Z_{t, z}^{\nu}(\tau) \in G(\tau)$ for all $\tau \in \mathcal{T}_{[t, T]}$. It follows from $\mathbf{Z 3}$ that $Z_{\theta, \xi}^{\nu}(\vartheta \vee \theta) \in G(\vartheta \vee \theta)$ for all $\vartheta \in \mathcal{T}_{[t, T]}$, where $\xi:=Z_{t, z}^{\nu}(\theta)$. Hence,

$$
1=\mathbb{P}\left[Z_{\theta, \xi}^{\nu}(\vartheta \vee \theta) \in G(\vartheta \vee \theta)\right]=\mathbb{E}\left[\mathbb{P}\left[Z_{\theta, \xi}^{\nu}(\vartheta \vee \theta) \in G(\vartheta \vee \theta) \mid(\theta, \xi)\right]\right] \quad \forall \vartheta \in \mathcal{T}_{[t, T]}
$$

Given $\vartheta \in \mathcal{T}_{[t, T]}$, set $J_{\vartheta}(\theta, \xi):=\mathbb{P}\left[Z_{\theta, \xi}^{\nu}(\vartheta \vee \theta) \in G(\vartheta \vee \theta) \mid(\theta, \xi)\right]$ and observe that the family $\left\{J_{\vartheta}(\theta, \xi), \vartheta \in\right.$ $\left.\mathcal{T}_{[t, T]}\right\}$ is directed downward. It follows that we can find a sequence $\left(\vartheta_{n}\right)_{n \geq 1}$ in $\mathcal{T}_{[t, T]}$ such that $\lim _{n} \downarrow J_{\vartheta_{n}}(\theta, \xi)=\operatorname{essinf}\left\{J_{\vartheta}(\theta, \xi), \vartheta \in \mathcal{T}_{[t, T]}\right\}$. The above equality combined with the monotone convergence theorem thus implies that

$$
1=\lim _{n} \downarrow \mathbb{E}\left[J_{\vartheta_{n}}(\theta, \xi)\right]=\mathbb{E}\left[\operatorname{ess} \inf _{\vartheta \in \mathcal{T}_{[t, T]}} J_{\vartheta}(\theta, \xi)\right],
$$

which, by Z22, implies that $\mathbb{P}\left[Z_{\bar{t}, \bar{z}}^{\nu}(\vartheta \vee \bar{t}) \in G(\vartheta \vee \bar{t})\right]=1$ for all $\vartheta \in \mathcal{T}_{[t, T]}$ for $\mu$-a.e. $(\bar{t}, \bar{z}) \in[t, T] \times \mathbb{R}^{d}$, where $\mu$ is the pull-back measure of $(\theta, \xi)$ under $\mathbb{P}$. This shows that $\bar{z} \in V(\bar{t})$ for $\mu$-a.e. $(\bar{t}, \bar{z}) \in[t, T] \times \mathbb{R}^{d}$ and therefore $\xi=Z_{t, z}^{\nu}(\theta) \in V(\theta) \mathbb{P}$-a.s. Since we already know that $Z_{t, z}^{\nu}(\tau) \in G(\tau)$ for all $\tau \in \mathcal{T}_{[t, T]}$, this shows that $z \in \bar{V}(t)$.

It remains to prove the opposite inclusion.

Lemma 2.2 $\bar{V}(t) \subset V(t)$.

Proof. We now fix $z \in \bar{V}(t)$ and $\nu \in \mathcal{A}$ such that

$$
Z_{t, z}^{\nu}(\theta \wedge \tau) \in G \stackrel{\tau, \theta}{\bigoplus} V \mathbb{P}-\text { a.s for all } \tau \in \mathcal{T}_{[t, T]} .
$$


1. We first work on the event set $\{\theta<\tau\}$. On this set, we have $Z_{t, z}^{\nu}(\theta) \in V(\theta)$ and therefore

$$
\left(\theta, Z_{t, z}^{\nu}(\theta)\right) \in D:=\{(t, z) \in S: z \in V(t)\}
$$

Let $\mathcal{B}_{D}$ denote the collection of Borel subsets of $D$. Applying Lemma 2.3 below to the measure induced by $\left(\theta, Z_{t, z}^{\nu}(\theta)\right)$ on $S$, we can construct a measurable map $\phi:\left(D, \mathcal{B}_{D}\right) \rightarrow\left(\mathcal{A}, \mathcal{B}_{\mathcal{A}}\right)$ such that

$$
Z_{\theta, Z_{t, z}^{\nu}(\theta)}^{\phi\left(\theta, Z_{t, z}^{\nu}(\theta)\right)}(\vartheta) \in G(\vartheta) \text { for all } \vartheta \in \mathcal{T}_{[\theta, T]},
$$

i.e.

$$
\phi\left(\theta, Z_{t, z}^{\nu}(\theta)\right) \in \mathcal{G}\left(\theta, Z_{t, z}^{\nu}(\theta)\right) .
$$

In view of $\mathbf{A 2}$, we can then find $\nu_{1} \in \mathcal{A}$ such that $\nu_{1}=\phi\left(\theta, Z_{t, z}^{\nu}(\theta)\right)$ on $[\theta, T] L e b \times \mathbb{P}$-a.e. It then follows from $\mathbf{A} 1$ that

$$
\hat{\nu}:=\nu 1_{[0, \theta)}+\nu_{1} 1_{[\theta, T]} \in \mathcal{A} .
$$

Moreover, according to $\mathbf{Z 3}$ and $\mathbf{Z 4}$, we have

$$
Z_{t, z}^{\hat{\nu}}(\tau)=Z_{\theta, Z_{t, z}^{\nu}(\theta)}^{\phi\left(\theta, Z_{t, z}^{\nu}(\theta)\right)}(\tau) \in G(\tau) \text { on }\{\theta<\tau\}
$$

2. Let $\hat{\nu}$ be defined as above and note that, by (2.1), we also have

$$
Z_{t, z}^{\hat{v}}(\tau)=Z_{t, z}^{\nu}(\tau) \in G \stackrel{\tau, \theta}{\bigoplus} V=G(\tau) \text { on }\{\tau \leq \theta\}
$$

3. Combining the two above steps shows that $\hat{\nu} \in \mathcal{G}(t, z)$ and therefore $z \in V(t)$.

It remains to prove the following result which was used in the previous proof.

Lemma 2.3 For any probability measure $\mu$ on $S$, there exists a Borel measurable function $\phi:\left(D, \mathcal{B}_{D}\right) \rightarrow$ $\left(\mathcal{A}, \mathcal{B}_{\mathcal{A}}\right)$ such that

$$
\varphi(t, z) \in \mathcal{G}(t, z) \text { for } \mu-\text { a.e. }(t, z) \in D .
$$

Proof. Set $B:=\{(t, z, \nu) \in S \times \mathcal{A}: \nu \in \mathcal{G}(t, z)\}$. It follows from Z5 that the map $(t, s, \nu) \in S \times \mathcal{A} \rightarrow$ $Z_{t, z}^{\nu}(r)$ is Borel measurable, for any $r \leq T$. Then, for any bounded continuous function $f$, the map $\psi_{f}^{r}:(t, s, \nu) \in S \times \mathcal{A} \rightarrow \mathbb{E}\left[f\left(Z_{t, z}^{\nu}(r)\right)\right]$ is Borel measurable. Since $G(r)$ is a Borel set, the map $1_{G(r)}$ is the limit of a sequence of bounded continuous functions $\left(f^{n}\right)_{n \geq 1}$. Therefore, $\psi_{1_{G(r)}}^{r}=\lim _{n \rightarrow \infty} \psi_{f^{n}}^{r}$ is a Borel function. This implies that $B^{r}$ is a Borel set, where, for $\theta \in \mathcal{T}_{[t, T]}$,

$$
B^{\theta}:=\left\{(t, s, \nu) \in S \times \mathcal{A}: \psi_{1_{G}}^{\theta}(t, z, \nu) \geq 1\right\}=\left\{(t, s, \nu) \in S \times \mathcal{A}: Z_{t, z}^{\nu}(\theta) \in G(\theta)\right\} .
$$

Since $B=\bigcap_{\theta \in \mathcal{T}_{[t, T]}} B^{\theta}$, appealing to the right-continuous assumption $\mathbf{G}$ and the right-continuity of $Z_{t, z}^{\nu}$, we deduce that $B=\bigcap_{r \leq T, r \in \mathbb{Q}} B^{r}$. This shows that $B$ is a Borel set and therefore an analytic subset of $S \times \mathcal{A}$, see [3].

Applying the Jankov-von Neumann Theorem (see [3] Proposition 7.49), we then deduce that there exists an analytically measurable function $\phi: D \rightarrow \mathcal{A}$ such that

$$
\varphi(t, z) \in \mathcal{G}(t, z) \text { for all }(t, z) \in D \text {. }
$$

Since an analytically measurable map is also universally measurable, the required result follows from Lemma 7.27 in [3]. 


\section{Application to American option pricing under constraints}

In this section, we explain how the American geometric dynamic programming principle of Theorem 2.1 can be used to relate the super-hedging price of an American option to a suitable classe of PDEs, in a Markovian setting.

More precisely, we shall consider a Brownian diffusion type financial market with portfolio strategies constrained to take values in a convex compact set. We note that the case of an unbounded set of controls can also be handled by following the ideas introduced in [7]. Similarly, jumps could be added to the dynamics without major difficulties, see [4].

On the other hand, we shall allow for a possibly degenerate diffusion coefficient, a case which as not been studied so far in this literature. We therefore take this opportunity to explain how it can be treated, which is of own interest and could be transposed to other hedging problems under constraints, e.g. for plain vanilla European options or for barrier options.

\subsection{The financial model}

From now on, we assume that the filtration $\mathbb{F}:=\left(\mathcal{F}_{t}\right)_{t \leq T}$ is generated by a standard $d$-dimensional Brownian motion $W$.

We consider a Markovian model of financial market composed of a non-risky asset, which price process is normalized to unity, and $d$ risky assets $X=\left(X^{1}, \ldots, X^{d}\right)$ whose dynamics are given by the stochastic differential equation

$$
d X(s)=\operatorname{diag}[X(s)] \mu(X(s)) d s+\operatorname{diag}[X(s)] \sigma(X(s)) d W_{s} .
$$

Here $\mu: \mathbb{R}^{d} \rightarrow \mathbb{R}^{d}$ and $\sigma: \mathbb{R}^{d} \rightarrow \mathbb{M}^{d}$, the set of $d \times d$-matrices. Time could be introduced in the coefficients without difficulties, under some additional regularity assumptions. We deliberately choose a time homogeneous dynamics to alleviate the notations.

Given $x \in(0, \infty)^{d}$, we denote by $X_{t, x}$ the solution of the above equation on $[t, T]$ satisfying $X_{t, x}(t)=x$. In order to guarantee the existence and uniqueness of a strong solution to (3.1), we assume that

$$
(\mu, \sigma) \text { is bounded and } x \in \mathbb{R}_{+}^{d} \mapsto(\operatorname{diag}[x] \sigma(x), \operatorname{diag}[x] \mu(x)) \text { is Lipschitz continuous. }
$$

Importantly, we do not assume that $\sigma$ is uniformly elliptic nor invertible, as in e.g. [2], [5] or [24].

A financial strategy is described by a $d$-dimensional predictable process $\pi=\left(\pi^{1}, \ldots, \pi^{d}\right)$. Each component stands for the proportion of the wealth invested in the corresponding risky asset. In this paper, we restrict to the case where the portfolio strategies are constrained to take values in a given compact convex set $K$ :

$$
\pi \in K \quad L e b \times d \mathbb{P} \text { - a.e, with } 0 \in K \subset \mathbb{R}^{d} \text { convex and compact. }
$$

We denote by $\mathcal{A}$ the set of such strategies.

To an initial capital $y \in \mathbb{R}_{+}$and a financial strategy $\pi$, we associate the induced wealth process $Y_{t, x, y}^{\pi}$ defined as the solution on $[t, T]$ of

$$
d Y(s)=Y(s) \pi_{s}^{\prime} \operatorname{diag}\left[X_{t, x}(s)\right]^{-1} d X_{t, x}(s)=Y(s) \pi_{s}^{\prime} \mu\left(X_{t, x}(s)\right) d s+Y(s) \pi_{s}^{\prime} \sigma\left(X_{t, x}(s)\right) d W_{s},
$$

with $Y(t)=y$. 


\subsection{The super-hedging problem and the dynamic programming principle}

The option is described by a locally bounded map $g$ defined on $[0, T] \times \mathbb{R}_{+}^{d}$ and satisfying

$$
g \geq 0 \text { and } g \text { is continuous on }[0, T] \times(0, \infty)^{d} .
$$

The buyer receives the payoff $g\left(\tau, X_{t, x}(\tau)\right)$ when he/she exercises the option at time $\tau \leq T$.

The super-hedging price is thus defined as

$$
v(t, x):=\inf \left\{y \in \mathbb{R}_{+}: \exists \pi \in \mathcal{A} \text { s.t } Y_{t, x, y}^{\pi}(\tau) \geq g\left(\tau, X_{t, x}(\tau)\right) \forall \tau \in \mathcal{T}_{[t, T]}\right\} .
$$

Remark 3.1 Note that $v(t, x)$ coincides with the lower bound of the set $\left\{y \in \mathbb{R}_{+} \quad: \quad(x, y) \in V(t)\right\}$ where

$$
V(t):=\left\{(x, y) \in(0, \infty)^{d} \times \mathbb{R}_{+}: \exists \pi \in \mathcal{A} \text { s.t. } Z_{t, x, y}^{\pi}(\tau) \in G(\tau) \forall \tau \in \mathcal{T}_{[t, T]}\right\},
$$

with $Z_{t, x, y}^{\pi}$ and $G$ defined as

$$
Z_{t, x, y}^{\pi}:=\left(X_{t, x}, Y_{t, x, y}^{\pi}\right) \text { and } G(t):=\left\{(x, y) \in \mathbb{R}_{+}^{d} \times \mathbb{R}_{+}: y \geq g(t, x)\right\} .
$$

Also notice that $\mathcal{A}$ satisfies the condition $\mathbf{A} \mathbf{1}$ of Section 2.1. Since $\mathcal{A}$ is a separable metric space, it also satisfies A2, see Remark 2.1 of Section 2.1. Moreover, Z1 - Z5 hold for $Z_{t, x, y}^{\pi}$ and the continuity assumption (3.4) implies that $G(t)$ satisfies the right-continuity condition $\mathbf{G}$ of Section 2.1.

It follows from the above Remark that the American geometric dynamic programming principle of Theorem 2.1 applies to $v$, compare with Example 2.1.

Corollary 3.1 Fix $(t, x, y) \in[0, T] \times(0, \infty)^{d} \times \mathbb{R}_{+}$.

(DP1). If $y>v(t, x)$, then there exists $\pi \in \mathcal{A}$ such that

$$
Y_{t, x, y}^{\pi}(\theta) \geq v\left(\theta, X_{t, x}(\theta)\right) \text { for all } \theta \in \mathcal{T}_{[t, T]} .
$$

(DP2) If $y<v(t, x)$, then $\exists \tau \in \mathcal{T}_{[t, T]}$ such that

$$
\mathbb{P}\left[Y_{t, x, y}^{\pi}(\theta \wedge \tau)>v\left(\theta, X_{t, x, y}(\theta)\right) 1_{\theta<\tau}+g\left(\tau, X_{t, x, y}(\tau)\right) 1_{\theta \geq \tau}\right]<1
$$

for all $\theta \in \mathcal{T}_{[t, T]}$ and $\pi \in \mathcal{A}$.

\subsection{PDE characterization of the super-replication price}

In this section, we show how the dynamic programming principle of Corollary 3.1 allows to provide a PDE characterization of the super-hedging price $v$. We start with a formal argument.

The first claim (DP1) of the geometric dynamic programming principle can be formally interpreted as follow. Set $y:=v(t, x)$ and assume that $v$ is smooth. Assuming that (DP1) of Corollary 3.1 holds for $y=v(t, x)$, we must then have, at least at a formal level, $d Y_{t, x, y}^{\pi}(t) \geq d v\left(t, X_{t, x}(t)\right)$, which can be achieved only if $\pi_{t}^{\prime} \mu(x) v(t, x)-\mathcal{L} v(t, x) \geq 0$ and $v(t, x) \pi^{\prime} \sigma(x)=(D v)^{\prime}(t, x) \operatorname{diag}[x] \sigma(x)$, where

$$
\mathcal{L} v(t, x):=\partial_{t} v(t, x)+(D v)^{\prime}(t, x) \operatorname{diag}[x] \mu(x)+\frac{1}{2} \operatorname{Trace}\left[\operatorname{diag}[x] \sigma(x) \sigma^{\prime}(x) \operatorname{diag}[x] D^{2} v(t, x)\right] .
$$

Moreover, we have by definition $v \geq g$ on $[0, T) \times(0, \infty)^{d}$. Thus, $v$ should be a supersolution of:

$$
\mathcal{H} \varphi(t, x):=\min \left\{\sup _{\pi \in \mathcal{N} \varphi(t, x)}\left(\pi^{\prime} \mu(x) \varphi(t, x)-\mathcal{L} \varphi(t, x)\right), \varphi-g\right\}=0,
$$


where, for a smooth function $\varphi$, we set $\mathcal{N} \varphi(t, x):=N(x, \varphi(t, x), D \varphi(t, x))$ with

$$
N(x, y, p):=\left\{\pi \in K: y \pi^{\prime} \sigma(x)=p^{\prime} \operatorname{diag}[x] \sigma(x)\right\}, \text { for }(x, y, p) \in(0, \infty)^{d} \times \mathbb{R}_{+} \times \mathbb{R}^{d},
$$

and we use the usual convention $\sup \emptyset=-\infty$.

Note that the supersolution property implies that $\mathcal{N} v \neq \emptyset$, in the viscosity sense. We shall show in Lemma 3.2 below that, for $(x, y, p) \in(0, \infty)^{d} \times \mathbb{R}_{+} \times \mathbb{R}^{d}$,

$$
N(x, y, p) \neq \emptyset \Longleftrightarrow M(x, y, p) \geq 0
$$

where

$$
M(x, y, p):=\inf _{\rho \in \tilde{K}_{x}}\left\{\delta_{x}(\rho) y-\rho^{\prime} \operatorname{diag}[x] p\right\}
$$

with

$$
\delta_{x}(\rho):=\sup \left\{\tilde{\pi}^{\prime} \rho, \tilde{\pi} \in K_{x}\right\} \quad, \quad K_{x}:=\left\{\tilde{\pi} \in \mathbb{R}^{d}: \tilde{\pi}^{\prime} \sigma(x)=\pi^{\prime} \sigma(x) \text { for some } \pi \in K\right\}
$$

and

$$
\tilde{K}_{x}:=\left\{\rho \in \mathbb{R}^{d}:|\rho|=1 \text { and } \delta_{x}(\rho)<\infty\right\} .
$$

Hence, $v$ should be a supersolution of

$$
\min \{\mathcal{H} \varphi, \mathcal{M} \varphi\}=0
$$

where $\mathcal{M} \varphi(t, x):=M(x, \varphi(t, x), D \varphi(t, x))$, the possible identity $M(x, v(t, x), D v(t, x))=0$ which is equivalent to $v(t, x)^{-1} \operatorname{diag}[x] D v(t, x) \in \partial K_{x}$, see [18], reflecting the fact that the constraint is binding.

Remark 3.2 Note that

$$
K=K_{x} \quad \text { and } \quad \delta_{x}(\rho)=\delta(\rho) \text { where } \delta(\rho):=\sup \left\{\pi^{\prime} \rho: \pi \in K\right\}, \text { when } \sigma(x) \text { is invertible. }
$$

Since $\mathcal{N} \varphi(t, x)$ is a singleton, when $\sigma$ is invertible, we then retrieve a formulation similar to [5] and [21].

Moreover, the minimality condition in the definition of $v$ should imply that $v$ actually solves (in some sense) the partial differential equation (3.8), with the usual convention $\sup \emptyset=-\infty$.

We shall first prove that $v$ is actually a viscosity solution of (3.8) in the sense of discontinuous viscosity solutions. In order to prove the subsolution property, we shall appeal to the additional regularity assumption:

Assumption 3.1 Fix $\left(x_{0}, y_{0}, p_{0}\right) \in(0, \infty)^{d} \times(0, \infty) \times \mathbb{R}^{d}$ such that $y_{0}^{-1} \operatorname{diag}\left[x_{0}\right] p_{0} \in \operatorname{int}\left(K_{x_{0}}\right)$. Set $\pi_{0} \in N\left(x_{0}, y_{0}, p_{0}\right)$. Then, for all $\varepsilon>0$, there exists an open neighborhood $\bar{B}$ of $\left(x_{0}, y_{0}, p_{0}\right)$ and a locally Lipschitz map $\hat{\pi}$ such that

$$
\left|\hat{\pi}\left(x_{0}, y_{0}, p_{0}\right)-\pi_{0}\right| \leq \varepsilon
$$

and

$$
\hat{\pi}(x, y, p) \in N(x, y, p) \text { on } \bar{B} \cap\left((0, \infty)^{d} \times(0, \infty) \times \mathbb{R}^{d}\right)
$$

Remark 3.3 In the case where $\sigma$ is invertible, it corresponds to Assumption 2.1 in [7].

Theorem 3.1 Assume that $v$ is locally bounded. Then, $v_{*}$ is a viscosity supersolution of (3.8) on $[0, T) \times(0, \infty)^{d}$. If moreover Assumption 3.1 holds, then $v^{*}$ is a viscosity subsolution of (3.8) on $[0, T) \times(0, \infty)^{d}$. 
Not surprisingly, the constraint $\mathcal{M} v \geq 0$ should propagate to the boundary point $t=T$ which implies that the boundary condition should be written in terms of the solution of the partial differential equation

$$
\min \{\phi(x)-g(T, x), \mathcal{M} \phi(x)\}=0 .
$$

Theorem 3.2 Assume that $v$ is locally bounded. Then, $v_{*}(T, \cdot)$ is a viscosity supersolution on $(0, \infty)^{d}$ of (3.10). Assume further that Assumption 3.1 holds, then $v^{*}(T, \cdot)$ is a viscosity subsolution on $(0, \infty)^{d}$ of (3.10).

We conclude by establishing a comparison result for (3.8)-(3.10) which implies that $v$ is continuous on $[0, T) \times(0, \infty)^{d}$, with a continuous extension to $[0, T] \times(0, \infty)^{d}$, and is the unique viscosity solution (3.8)-(3.10) in a suitable class of functions. To this purpose, we shall need the following additional assumptions:

\section{Assumption 3.2}

(i) There exists $\bar{\gamma} \in K \cap[0, \infty)^{d}$ and $\lambda>1$ such that $\lambda \bar{\gamma} \in K_{x}$ for all $x \in(0, \infty)^{d}$ and such that

(ii) there exists a constant $C>0$ such that $|g(t, x)| \leq C\left(1+x^{\bar{\gamma}}\right)$ for all $(t, x) \in[0, T] \times(0, \infty)^{d}$.

(iii) There exists $c_{K}>0$ such that $\delta_{x}(\rho) \geq c_{K}$ for all $x \in(0, \infty)^{d}$ and $\rho \in \tilde{K}_{x}$.

(iv) There exists $C>0$ such that, for all $x, y \in(0, \infty)^{d}$ and $\rho \in \tilde{K}_{x}$, we can find $\tilde{\rho} \in \tilde{K}_{y}$ satisfying $|\rho-\tilde{\rho}| \leq C|x-y|$ and $\delta_{y}(\tilde{\rho})-\delta_{x}(\rho) \leq \epsilon(x, y)$, where $\epsilon$ is a continuous map satisfying $\epsilon(z, z)=0$ for all $z \in(0, \infty)^{d}$.

(v) Either

(v.a.) There exists $L>0$ such that, for all $(x, \bar{x}, y, \bar{y}, p, \bar{p}) \in(0, \infty)^{2 d} \times \mathbb{R}_{+}^{2} \times \mathbb{R}^{2 d}: \pi \in N(x, y, p) \neq \emptyset$, $N(\bar{x}, \bar{y}, \bar{p}) \neq \emptyset$ and $|x-\bar{x}| \leq L^{-1} \Longrightarrow \exists \bar{\pi} \in N(\bar{x}, \bar{y}, \bar{p})$ s.t.

$$
\left|y \pi^{\prime} \mu(x)-\bar{y} \bar{\pi}^{\prime} \mu(\bar{x})\right| \leq L\left|p^{\prime} \operatorname{diag}[x] \mu(x)-\bar{p}^{\prime} \operatorname{diag}[\bar{x}] \mu(\bar{x})\right| .
$$

or

(v.b.) For all $p, q \in \mathbb{R}^{d}$ and $x \in(0, \infty)^{d}: p^{\prime} \sigma(x)=q^{\prime} \sigma(x) \Longrightarrow p^{\prime} \mu(x)=q^{\prime} \mu(x)$.

Theorem 3.3 Let Assumptions 3.1 and 3.2 hold. Then, $v^{*}=v_{*}$ is continuous on $[0, T] \times(0, \infty)^{d}$ and is the unique viscosity solution of (3.8)-(3.10) in the class of non-negative functions satisfying the growth condition (ii) of Assumption 3.2.

Remark 3.4 1. The first condition holds whenever $\lambda \bar{\gamma} \in K$.

2. The condition (ii) implies that

$$
\exists C>0 \text { s.t. }|v(t, x)| \leq C\left(1+x^{\bar{\gamma}}\right) \quad \forall(t, x) \in[0, T] \times(0, \infty)^{d} .
$$

Indeed, let $\pi \in \mathcal{A}$ be defined by $\pi_{s}=\bar{\gamma}$ for all $t \leq s \leq T$. Since $\sigma$ is bounded, one easily checks from the dynamics of the processes $X_{t, x}$ and $Y_{t, x, 1}^{\pi}$ that

$$
1+\prod_{i=1}^{d}\left(X_{t, x}^{i}(u)\right)^{\bar{\gamma}^{i}} \leq C\left(1+\prod_{i=1}^{d}\left(x^{i}\right)^{\bar{\gamma}^{i}}\right) Y_{t, x, 1}^{\pi}(u) \quad \text { for all } u \in[t, T],
$$

where $C>0$ depends only on $|\bar{\gamma}|$ and the bound on $|\sigma|$. Then, after possibly changing the value of the constant $C$, (ii) of Assumption 3.2 implies

$$
g\left(u, X_{t, x}(u)\right) \leq C\left(1+x^{\bar{\gamma}}\right) Y_{t, x, 1}^{\pi}(u) \quad \text { for all } u \in[0, T] .
$$

Since $y Y_{t, x, 1}^{\pi}=Y_{t, x, y}^{\pi}$ for $y>0$, we deduce (3.11) from the last inequality. 
3. The condition (iii) is implied by $0 \in \operatorname{int}(K)$. Indeed, if $0 \in \operatorname{int}(K)$, then $\delta_{x} \geq \delta$ where the later is uniformly strictly positive, see [18].

4. The condition (iv) is trivially satisfied if $\delta_{x}=\delta$ for all $x \in(0, \infty)^{d}$, which is the case when $\sigma$ is invertible.

5. The condition (v) is trivially satisfied when $\sigma$ is invertible. The condition (v.b.) is natural in the case $0 \in \operatorname{int}(K)$ as, in this case, it is equivalent to $\pi^{\prime} \sigma(x)=0 \Rightarrow \pi^{\prime} \mu(x) \leq 0$ for all $\pi \in K$, which is intimately related to the minimal no-arbitrage condition: $\pi \in \mathcal{A}$ and $Y_{t, x, y} \pi^{\prime} \sigma\left(X_{t, x}\right)=0$ Leb $\times \mathbb{P}$-a.e. on $[t, T] \Rightarrow Y_{t, x, y} \pi^{\prime} \mu\left(X_{t, x}\right) \leq 0$ Leb $\times \mathbb{P}$-a.e. on $[t, T]$.

\subsection{The "face-lift" phenomenon}

When $\sigma$ is invertible, it can be shown under mild assumptions, see Section 3.5.4 below, that the unique viscosity solution of $(3.10)$, in a suitable class, is given by $\hat{g}(T, \cdot)$ where

$$
\hat{g}(t, x):=\sup _{\rho \in \mathbb{R}^{d}} e^{-\delta(\rho)} g\left(t, x e^{\rho}\right) .
$$

A standard comparison theorem, see Section 3.5.4 below, then implies that the boundary condition of Theorem 3.2 can actually be written in $v_{*}(T, \cdot)=v^{*}(T, \cdot)=\hat{g}(T, \cdot)$. This is the so-called "face-lift" procedure which was already observed by [8] in the context of European option pricing, see also [2], [4], [5] or [11]. Similarly, one could replace $g$ by $\hat{g}$ in the definition of $\mathcal{H} \varphi$ by using (3.7).

In our general context, where $\sigma$ is not assumed to be invertible anymore, the solution of the PDE

$$
\min \left\{\phi(x)-g\left(t_{0}, x\right), \mathcal{M} \phi(x)\right\}=0
$$

has a more complex structure. Standard optimal control arguments actually show that it should be related to the deterministic control problem

$$
\check{g}\left(t_{0}, x\right):=\sup _{(\rho, \tau) \in L_{1}^{0}(L e b) \times \mathbb{R}_{+}} e^{-\int_{0}^{\tau} \delta_{\chi_{x}^{\rho}(s)}\left(\rho_{s}\right) d s} g\left(t_{0}, \chi_{x}^{\rho}(\tau)\right)
$$

where $L_{1}^{0}(L e b)$ denotes the set of measurable functions $\rho=\left(\rho_{s}\right)_{s \geq 0}$ on $\mathbb{R}_{+}$satisfying $\left|\rho_{t}\right|=1$ for all $t \geq 0$ and, for $\rho \in L_{1}^{0}(L e b)$, the process $\chi_{x}^{\rho}$ is the solution of

$$
\chi(t)=x+\int_{0}^{t} \operatorname{diag}[\chi(s)] \rho_{s} d s, \quad t \geq 0 .
$$

Note that, in the case where $\sigma$ is invertible, (3.9) implies that it can be rewritten in

$$
\sup _{(\rho, \tau) \in L_{1}^{0}(L e b) \times \mathbb{R}_{+}} e^{-\int_{0}^{\tau} \delta\left(\rho_{s}\right) d s} g\left(t_{0}, \chi_{x}^{\rho}(\tau)\right)
$$

which value function is easily seen to coincide with $\hat{g}\left(t_{0}, \cdot\right)$ by using the fact that $\delta$ is convex and homegenous of degree one, and $g \geq 0$.

We now make precise the above discussion in the two following Corollaries. The first one actually states that $g$ can be replaced by $\check{g}$ in the definition of $\mathcal{H}$ and in the terminal condition.

Corollary 3.2 Let Assumption 3.1 and (i)-(iv) of Assumption 3.2 hold. Assume further that:

(i) For each $x \in(0, \infty)^{d}$, the map $t \in[0, T] \mapsto \check{g}(t, x)$ is continuous.

(ii) The map $x \in(0, \infty)^{d} \mapsto \sup \left\{\delta_{x}(\rho), \rho \in \tilde{K}_{x}\right\}$ is locally bounded.

Then, $v$ is a discontinuous viscosity solution on $[0, T) \times(0, \infty)^{d}$ of

$$
\min \left\{\sup _{\pi \in \mathcal{N} \varphi(t, x)}\left(\pi^{\prime} \mu(x) \varphi(t, x)-\mathcal{L} \varphi(t, x)\right), \mathcal{M} \varphi(t, x), \varphi(t, x)-\check{g}(t, x)\right\}=0
$$


and satisfies the boundary condition

$$
v_{*}(T, \cdot)=v^{*}(T, \cdot)=\check{g}(T, \cdot) .
$$

If moreover, (v) of Assumption 3.2 hold, then it is the unique viscosity solution of (3.12)-(3.13), in the class of non-negative functions satisfying the growth condition (3.11).

In the case where $\sigma$ is invertible, the above discussion already shows that $\hat{g}=\check{g}$. If moreover $\mu$ and $\sigma$ are constant, we can actually interpret the super-hedging price as the price of an American option with payoff $\hat{g}$, without taking the portfolio constraints into account. This phenomenon was already observed for plain vanilla or barrier european options, see e.g. [5] and [8]. It comes from the fact that, when the parameters are constant, the gradient constraint imposed at $T$ by the terminal condition $v(T, \cdot)=\hat{g}$ propagates into the domain. It is therefore automatically satisfied and we retrieve the result of Corollary 1 in $[8]$.

Corollary 3.3 Let the conditions of Theorem 3.3 hold. Assume further that $\mu$ and $\sigma$ are constant and that $\sigma$ is invertible. Then, $\check{g}=\hat{g}$ and

$$
v(t, x)=\sup _{\tau \in \mathcal{T}_{[t, T]}} \mathbb{E}^{\mathbb{Q}}\left[\hat{g}\left(\tau, X_{t, x}(\tau)\right)\right]
$$

where $\mathbb{Q} \sim \mathbb{P}$ is defined by

$$
\frac{d \mathbb{Q}}{d \mathbb{P}}:=e^{-\frac{1}{2}\left|\sigma^{-1} \mu\right|^{2} T+\left(\sigma^{-1} \mu\right)^{\prime} W_{T}} .
$$

Remark 3.5 Since $\sigma$ is constant and invertible, the condition that $\mu$ is constant could be relaxed, under mild assumptions, by performing a suitable initial change of measure.

\subsection{Proof of the PDE characterization}

From now on, we assume that $v$ is locally bounded.

\subsubsection{The supersolution property}

We start with the supersolution property of Theorem 3.1 and Theorem 3.2.

Lemma 3.1 The map $v_{*}$ is a viscosity supersolution of $\mathcal{H} \varphi=0$ on $[0, T) \times(0, \infty)^{d}$.

Proof. Note that $v \geq g$ by definition. Since $g$ is continuous, this implies that $v_{*} \geq g$. It thus suffices to show that $v_{*}$ is a supersolution of

$$
\sup _{\pi \in \mathcal{N} \varphi(t, x)}\left(\pi^{\prime} \mu(x) \varphi(t, x)-\mathcal{L} \varphi(t, x)\right)=0 .
$$

The proof follows from similar arguments as in [21], the main difference comes from the fact that $\sigma$ is not assumed to be non-degenate which only modifies the terminal argument of the above paper. We therefore only sketch the proof and focus on the main difference. Fix $\left(t_{0}, x_{0}\right) \in[0, T) \times(0, \infty)^{d}$ and let $\varphi$ be a smooth function such that $\left(t_{0}, x_{0}\right)$ achieves a strict minimum of $v_{*}-\varphi$ on $[0, T) \times(0, \infty)^{d}$ satisfying $\left(v_{*}-\varphi\right)\left(t_{0}, x_{0}\right)=0$. Let $\left(t_{n}, x_{n}\right)_{n \geq 1}$ be a sequence in $[0, T) \times(0, \infty)^{d}$ that converges to $\left(t_{0}, x_{0}\right)$ and such that

$$
v\left(t_{n}, x_{n}\right) \rightarrow v_{*}\left(t_{0}, x_{0}\right) \text { as } n \rightarrow \infty
$$


We have

$$
y_{0}:=\varphi\left(t_{0}, x_{0}\right)=v_{*}\left(t_{0}, x_{0}\right)=\lim _{n \rightarrow \infty} v\left(t_{n}, x_{n}\right)
$$

Set

$$
y_{n}:=v\left(t_{n}, x_{n}\right)+\frac{1}{n} .
$$

Since $y_{n}>v\left(t_{n}, x_{n}\right)$, it follows from (DP1) of Corollary 3.1 that we can fin $\pi_{n} \in \mathcal{A}$ such that, for any stopping time $\tau_{n} \in \mathcal{T}_{\left[t_{n}, T\right]}$, we have

$$
Y_{t_{n}, x_{n}, y_{n}}^{\pi_{n}}\left(\tau_{n}\right) \geq v\left(\tau_{n}, X_{t_{n}, x_{n}}\left(\tau_{n}\right)\right) .
$$

Since $v \geq v_{*} \geq \varphi$, it follows that

$$
Y_{t_{n}, x_{n}, y_{n}}^{\pi_{n}}\left(\tau_{n}\right) \geq v\left(\tau_{n}, X_{t_{n}, x_{n}}\left(\tau_{n}\right)\right) \geq \varphi\left(\tau_{n}, X_{t_{n}, x_{n}}\left(\tau_{n}\right)\right) .
$$

Set $Y_{n}:=Y_{t_{n}, x_{n}, y_{n}}^{\pi_{n}}, X_{n}:=X_{t_{n}, x_{n}}$. It follows from the previous inequality and Ito's Lemma that

$$
\begin{aligned}
0 \leq & y_{n}+\int_{t_{n}}^{\tau_{n}} Y_{n}(s) \pi_{n}^{\prime}(s) \sigma\left(X_{n}(s)\right) d W_{s}+\int_{t_{n}}^{\tau_{n}} Y_{n}(s) \pi_{n}^{\prime}(s) \mu\left(X_{n}(s)\right) d s \\
& -\varphi\left(t_{n}, x_{n}\right)-\int_{t_{n}}^{\tau_{n}} \mathcal{L} \varphi\left(s, X_{n}(s)\right) d s-\int_{t_{n}}^{\tau_{n}}(D \varphi)^{\prime}\left(s, X_{n}(s)\right) \operatorname{diag}\left[X_{n}(s)\right] \sigma\left(X_{n}(s)\right) d W_{s}
\end{aligned}
$$

which can be written as

$$
0 \leq \beta_{n}+\int_{t_{n}}^{\tau_{n}} Y_{n}(s) \pi_{n}^{\prime}(s) \mu\left(X_{n}(s)\right)-\mathcal{L} \varphi\left(s, X_{n}(s)\right) d s+\int_{t_{n}}^{\tau_{n}} \psi\left(s, X_{n}(s), Y_{n}(s), \pi_{n}(s)\right) d W_{s},
$$

where $\beta_{n}:=y_{n}-\varphi\left(t_{n}, x_{n}\right)$ and

$$
\psi:(s, x, y, \pi) \rightarrow\left(y \pi^{\prime}-(D \varphi)^{\prime}(s, x) \operatorname{diag}[x]\right) \sigma(x) .
$$

By choosing a suitable sequence of stopping times $\left(\tau_{n}\right)_{n}$, introducing a well-chose sequence of change of measures as in Section 4.1 of [21] and using the Lipschitz continuity assumption (3.2) and the fact that $K$ is convex and compact, we deduce from the previous inequality and exactly the same arguments as in [21] that, for all $\kappa>0$,

$$
0 \leq \sup _{\pi \in K}\left(\varphi\left(t_{0}, x_{0}\right) \pi^{\prime} \mu\left(x_{0}\right)-\mathcal{L} \varphi\left(t_{0}, x_{0}\right)+\kappa\left|\psi\left(t_{0}, x_{0}, y_{0}, \pi\right)\right|^{2}\right) .
$$

Recalling that $K$ is compact and $\psi$ is continuous, we obtain by sending $\kappa$ to $\infty$ that

$$
\varphi\left(t_{0}, x_{0}\right) \pi^{\prime} \mu\left(x_{0}\right)-\mathcal{L} \varphi\left(t_{0}, x_{0}\right) \geq 0 \text { and }\left|\psi\left(t_{0}, x_{0}, y_{0}, \pi\right)\right|^{2}=0 \text { for some } \pi \in K .
$$

Noting that

$$
0=\psi\left(t_{0}, x_{0}, y_{0}, \pi\right)=\left(y_{0} \pi^{\prime}-(D \varphi)^{\prime}\left(t_{0}, x_{0}\right) \operatorname{diag}\left[x_{0}\right]\right) \sigma\left(x_{0}\right) \Rightarrow \pi \in \mathcal{N} \varphi\left(t_{0}, x_{0}\right),
$$

we finally obtain

$$
\sup _{\pi \in \mathcal{N} \varphi\left(t_{0}, x_{0}\right)}\left(\varphi\left(t_{0}, x_{0}\right) \pi^{\prime} \mu\left(x_{0}\right)-\mathcal{L} \varphi\left(t_{0}, x_{0}\right)\right) \geq 0
$$

As explained in Section 3.3, we now use the fact that $\mathcal{N} \varphi(t, x) \neq \emptyset$ if and only if $\mathcal{M} \varphi(t, x) \geq 0$.

Lemma 3.2 Fix $(x, y, p) \in(0, \infty)^{d} \times \mathbb{R}_{+} \times \mathbb{R}^{d}$. Then, $N(x, y, p) \neq \emptyset$ if and only if $M(x, y, p) \geq 0$. If moreover $y>0$, then $y^{-1} \operatorname{diag}[x] p \in \operatorname{int}\left(K_{x}\right)$ if and only if $M(x, y, p)>0$. 
Proof. For $y>0, N(x, y, p) \neq \emptyset \Leftrightarrow y^{-1} \operatorname{diag}[x] p \in K_{x} \Leftrightarrow M(x, y, p) \geq 0$ since $K_{x}$ is a closed convex set, see [18], and similarly, for $y>0, y^{-1} \operatorname{diag}[x] p \in \operatorname{int}\left(K_{x}\right)$ if and only if $M(x, y, p)>0$. We now consider the case $y=0$. Since $0 \in K \subset K_{x}$, we have $\delta_{x} \geq 0$. Hence, $N(x, 0, p) \neq \emptyset \Leftrightarrow 0 \sigma(x)=p^{\prime} \operatorname{diag}[x] \sigma(x) \Leftrightarrow$ $\varepsilon^{-1} p^{\prime} \operatorname{diag}[x] \in K_{x}$ for each $\varepsilon>0 \Leftrightarrow M(x, \varepsilon, p) \geq 0$ for each $\varepsilon>0 \Leftrightarrow M(x, 0, p) \geq 0$.

As a corollary of Lemma 3.1 and the previous Lemma, we obtain:

Corollary 3.4 The map $v_{*}$ is a viscosity supersolution of $\mathcal{M} \varphi=0$ on $[0, T) \times(0, \infty)^{d}$.

We now turn to the boundary condition at $t=T$.

Lemma 3.3 The map $v_{*}\left(T, \dot{)}\right.$ is a viscosity supersolution of $\min \left\{v_{*}(T, \cdot)-g(T, \cdot), \mathcal{M} \phi\right\}=0$ on $(0, \infty)^{d}$.

Proof. The fact that $v_{*}(T, \cdot) \geq g(T, \cdot)$ follows from the continuity of $g$ and the fact that $v \geq g$ on $[0, T) \times(0, \infty)^{d}$ by definition. Let $\phi$ be a smooth function and $x_{0} \in(0, \infty)^{d}$ be such that $x_{0}$ achieves a strict minimum of $v_{*}(T, \cdot)-\phi$ and $v_{*}\left(T, x_{0}\right)-\phi\left(x_{0}\right)=0$. Let $\left(s_{n}, \xi_{n}\right)_{n}$ be a sequence in $[0, T) \times(0, \infty)^{d}$ satisfying :

$$
\left(s_{n}, \xi_{n}\right) \longrightarrow\left(T, x_{0}\right), s_{n}<T \text { and } v_{*}\left(s_{n}, \xi_{n}\right) \longrightarrow v_{*}\left(T, x_{0}\right) .
$$

For all $n \in \mathbb{N}$ and $k>0$, we define :

$$
\varphi_{n}^{k}(t, x):=\phi(x)-\frac{k}{2}\left|x-x_{0}\right|^{2}+k \frac{T-t}{T-s_{n}} .
$$

Notice that $0 \leq(T-t)\left(T-s_{n}\right)^{-1} \leq 1$ for $t \in\left[s_{n}, T\right]$, and therefore :

$$
\lim _{k \rightarrow 0} \limsup _{n \rightarrow \infty} \sup _{(t, x) \in\left[s_{n}, T\right] \times B_{r}\left(x_{0}\right)}\left|\varphi_{n}^{k}(t, x)-\phi(x)\right|=0,
$$

where $r>0$ is such that $B_{r}\left(x_{0}\right) \subset(0, \infty)^{d}$. Next, let $\left(t_{n}^{k}, x_{n}^{k}\right)$ be a sequence of local minimizers of $v_{*}-\varphi_{n}^{k}$ on $\left[s_{n}, T\right] \times B_{r}\left(x_{0}\right)$ and set $e_{n}^{k}:=\left(v_{*}-\varphi_{n}^{k}\right)\left(t_{n}^{k}, x_{n}^{k}\right)$. Following line by line the arguments of the proof of Lemma 20 in [4], one easily checks that, after possibly passing to a subsequence :

$$
\begin{aligned}
& \text { for all } k>0, \quad\left(t_{n}^{k}, x_{n}^{k}\right) \longrightarrow\left(T, x_{0}\right), \\
& \text { for all } k>0, \quad t_{n}^{k}<T \text { for sufficiently large } n, \\
& v_{*}\left(t_{n}^{k}, x_{n}^{k}\right) \longrightarrow v_{*}\left(T, x_{0}\right)=\phi\left(x_{0}\right) \text { as } n \rightarrow \infty \text { and } k \rightarrow 0 .
\end{aligned}
$$

Notice that (3.17) and a standard diagonalization argument implies that we may assume that $x_{n}^{k} \in$ $\left(B_{r}\left(x_{0}\right)\right)$ for all $n \geq 1$ and $k>0$. It then follows from (3.18) that, for all $k>0,\left(t_{n}^{k}, x_{n}^{k}\right)$ is a sequence of local minimizers of $v_{*}-\varphi_{n}^{k}$ on $\left[s_{n}, T\right) \times B_{r}\left(x_{0}\right)$. Also, notice that (3.16), (3.17) and (3.19) imply

$$
\begin{aligned}
& \text { for all } k>0, D \varphi_{n}^{k}\left(t_{n}^{k}, x_{n}^{k}\right)=D \phi\left(x_{n}^{k}\right)-k\left(x_{n}^{k}-x_{0}\right) \rightarrow D \phi\left(x_{0}\right), \\
& \text { and } \lim _{k \rightarrow 0} \lim _{n \rightarrow \infty} e_{n}^{k}=0 .
\end{aligned}
$$

It then follows from Theorem 3.1, recall the convention $\sup \emptyset=-\infty,(3.18)$ and the fact that $\left(t_{n}^{k}, x_{n}^{k}\right)$ is a local minimizer for $v_{*}-\varphi_{n}^{k}$ that, for sufficiently large $n$, we can find $\pi_{n}^{k} \in K$ such that

$$
v_{*}\left(t_{n}^{k}, x_{n}^{k}\right)\left(\pi_{n}^{k}\right)^{\prime} \sigma\left(x_{n}^{k}\right)=D \varphi_{n}^{k}\left(t_{n}^{k}, x_{n}^{k}\right)^{\prime} \operatorname{diag}\left[x_{n}^{k}\right] \sigma\left(x_{n}^{k}\right) .
$$

Since $K$ is compact, we can assume that $\pi_{n}^{k} \rightarrow \pi \in K$ as $n \rightarrow \infty$ and then $k \rightarrow 0$. Taking the limit as $n \rightarrow \infty$ and then as $k \rightarrow 0$ in the previous inequality, and using (3.17), (3.19), (3.20), as well as the continuity of $x \mapsto \operatorname{diag}[x] \sigma(x)$ thus implies that

$$
v_{*}\left(T, x_{0}\right) \pi^{\prime} \sigma\left(x_{0}\right)=D \phi\left(x_{0}\right)^{\prime} \operatorname{diag}\left[x_{0}\right] \sigma\left(x_{0}\right) .
$$

Appealing to Lemma 3.2 then implies the required result. 


\subsubsection{The subsolution property}

We now turn to the subsolution property of Theorem 3.1 and Theorem 3.2.

Lemma 3.4 Under Assumption 3.1, the map $v^{*}$ is a viscosity subsolution of $\min \{\mathcal{H} \varphi, \mathcal{M} \varphi\}=0$ on $[0, T) \times(0, \infty)^{d}$.

Proof. Fix $\left(t_{0}, x_{0}\right) \in[0, T) \times(0, \infty)^{d}$ and let $\varphi$ be a smooth function such that $\left(t_{0}, x_{0}\right)$ achieves a strict maximum of $v^{*}-\varphi$ on $[0, T) \times(0, \infty)^{d}$ satisfying $\left(v^{*}-\varphi\right)\left(t_{0}, x_{0}\right)=0$. We assume that

$$
\min \left\{\sup _{\pi \in \mathcal{N} \varphi\left(t_{0}, x_{0}\right)}\left(\varphi\left(t_{0}, x_{0}\right) \pi^{\prime} \mu\left(x_{0}\right)-\mathcal{L} \varphi\left(t_{0}, x_{0}\right)\right), \varphi\left(t_{0}, x_{0}\right)-g\left(t_{0}, x_{0}\right), \mathcal{M} \varphi\left(t_{0}, x_{0}\right)\right\}:=2 \varepsilon>0,
$$

and work towards a contradiction. Note that $v^{*}\left(t_{0}, x_{0}\right)=\varphi\left(t_{0}, x_{0}\right)>0$ since $g \geq 0$. In view of Lemma 3.2 , this implies that $\varphi\left(t_{0}, x_{0}\right)^{-1} \operatorname{diag}\left[x_{0}\right] D \varphi\left(t_{0}, x_{0}\right) \in \operatorname{int}\left(K_{x_{0}}\right)$. It then follows from Assumption 3.1 that we can find $r>0$ and a Lipschitz continuous map $\hat{\pi}$ such that

$$
\min \left\{y \hat{\pi}(x, y, D \varphi(t, x))^{\prime} \mu(x)-\mathcal{L} \varphi(t, x), y-g(t, x)\right\}>\varepsilon
$$

and

$$
\hat{\pi}(x, y, D \varphi(t, x)) \in N(x, y, D \varphi(t, x)) \quad \text { for }(t, x, y) \in B_{r}\left(t_{0}, x_{0}\right) \times B_{r}(\varphi(t, x)) .
$$

Let $\left(t_{n}, x_{n}\right)_{n}$ be a sequence in $B_{r}\left(t_{0}, x_{0}\right)$ such that $v\left(t_{n}, x_{n}\right) \rightarrow v^{*}\left(t_{0}, x_{0}\right)$ and set $y_{n}:=v\left(t_{n}, x_{n}\right)-n^{-1}$ so that $y_{n}>0$ for $n$ large enough. Without loss of generality, we can assume that $y_{n} \in B_{r}\left(\varphi\left(t_{n}, x_{n}\right)\right)$ for each $n$. Let $\left(X^{n}, Y^{n}\right)$ denote the solution of (3.1) and (3.3) associated to the Markovian control $\hat{\pi}\left(X^{n}, Y^{n}, D \varphi\left(\cdot, X^{n}\right)\right)$ and the initial conditions $\left(X^{n}\left(t_{n}\right), Y^{n}\left(t_{n}\right)\right)=\left(x_{n}, y_{n}\right)$. Note that these processes are well defined on $\left[t_{n}, \tau_{n}\right]$ where

$$
\tau_{n}:=\inf \left\{t \geq t_{n}:\left(t, X^{n}(t)\right) \notin B_{r}\left(t_{0}, x_{0}\right) \text { or }\left|Y^{n}(t)-\varphi\left(t, X^{n}(t)\right)\right| \geq r\right\} .
$$

Moreover, it follows from the definition of $\left(t_{0}, x_{0}\right)$ as a strict maximum point of $v^{*}-\varphi$ that

$$
\left(v^{*}-\varphi\right)\left(\tau_{n}, X^{n}\left(\tau_{n}\right)\right)<-\zeta \quad \text { or }\left|Y^{n}\left(\tau_{n}\right)-\varphi\left(\tau_{n}, X^{n}\left(\tau_{n}\right)\right)\right| \geq r
$$

for some $\zeta>0$. Since $v^{*} \leq \varphi$, applying Itô's Lemma to $Y^{n}-\varphi\left(\cdot, X^{n}\right)$, recalling (3.22) and using a standard comparison Theorem for stochastic differential equations shows that

$$
Y^{n}\left(\tau_{n}\right)-v\left(\tau_{n}, X^{n}\left(\tau_{n}\right)\right) \geq Y^{n}\left(\tau_{n}\right)-\varphi\left(\tau_{n}, X^{n}\left(\tau_{n}\right)\right) \geq r \quad \text { on }\left\{\left|Y^{n}\left(\tau_{n}\right)-\varphi\left(\tau_{n}, X^{n}\left(\tau_{n}\right)\right)\right| \geq r\right\} .
$$

The same arguments combined with (3.23) also implies that

$$
Y^{n}\left(\tau_{n}\right)-v\left(\tau_{n}, X^{n}\left(\tau_{n}\right)\right) \geq y_{n}-\varphi\left(t_{n}, x_{n}\right)+\zeta \quad \text { on }\left\{\left|Y^{n}\left(\tau_{n}\right)-\varphi\left(\tau_{n}, X^{n}\left(\tau_{n}\right)\right)\right|<r\right\} .
$$

Since $y_{n}-\varphi\left(t_{n}, x_{n}\right) \rightarrow 0$, combining the two last assertions shows that $Y^{n}\left(\tau_{n}\right)-v\left(\tau_{n}, X^{n}\left(\tau_{n}\right)\right)>0$ for $n$ large enough. Moreover, it follows from (3.22) that $Y^{n}>g\left(\cdot, X^{n}\right)$ on $\left[t_{n}, \tau_{n}\right]$. Since $y_{n}<v\left(t_{n}, x_{n}\right)$, this contradicts (DP2) of Corollary 3.1.

Lemma 3.5 Let Assumption 3.1 hold. Fix $x_{0} \in(0, \infty)^{d}$ and assume that there exists a smooth function $\phi$ such that $\mathcal{M} \phi\left(x_{0}\right)>0$ and $x_{0}$ achieves a strict local maximum of $v^{*}(T, \cdot)-\phi$. Then, $v^{*}\left(T, x_{0}\right) \leq$ $g\left(T, x_{0}\right)$.

Proof. Without loss of generality, we may assume that $v^{*}\left(T, x_{0}\right)-\phi\left(x_{0}\right)=0$. Assume that $v^{*}\left(T, x_{0}\right)>$ $g\left(T, x_{0}\right)$. Note that this implies that $\phi\left(x_{0}\right)>0$. Since $g$ is continuous and $\mathcal{M} \phi\left(x_{0}\right)>0$, it follows from Lemma 3.2 and Assumption 3.1 that we can find $r, \eta>0$ and a Lipschitz continuous map $\hat{\pi}$ such that $\hat{\pi}(x, y, D \varphi(t, x)) \in N(x, y, D \varphi(t, x)), \varphi(t, x)-g(t, x) \geq \eta \quad$ for $(t, x, y) \in[T-r, T] \times B_{r}\left(x_{0}\right) \times B_{r}(\varphi(t, x))$ 
where $\varphi(t, x):=\phi(x)+\left|x-x_{0}\right|^{4}+\sqrt{T-t}$ for $(t, x) \in[0, T] \times(0, \infty)^{d}$. Since $\partial_{t} \varphi \rightarrow-\infty$ as $t \rightarrow T$ and $K$ is compact, we also have, after possibly changing $r>0$,

$$
y \hat{\pi}(x, y, D \varphi(t, x))^{\prime} \mu(x)-\mathcal{L} \varphi(t, x) \geq \eta \quad \text { for }(t, x, y) \in[T-r, T] \times B_{r}\left(x_{0}\right) \times B_{r}(\varphi(t, x)) .
$$

Let $\left(t_{n}, x_{n}\right)_{n}$ be a sequence in $[0, T] \times(0, \infty)^{d}$ such that $v\left(t_{n}, x_{n}\right) \rightarrow v^{*}\left(t_{n}, x_{n}\right)$ and let $\left(\hat{t}_{n}, \hat{x}_{n}\right)$ be a strict maximum point of $v^{*}-\varphi$ on $\left[t_{n}-n^{-1}, T\right] \times(0, \infty)^{d}$. Arguing as in Section 5.2 of [4], one easily checks that $\hat{t}_{n}<T$ and that $\left(\hat{t}_{n}, \hat{x}_{n}\right) \rightarrow\left(t_{0}, x_{0}\right)$. Let $\left(X^{n}, Y^{n}\right)$ denote the solution of (3.1) and (3.3) associated to the Markovian control $\hat{\pi}\left(X^{n}, Y^{n}, D \varphi\left(\cdot, X^{n}\right)\right)$ and the initial conditions $\left(X^{n}\left(\hat{t}_{n}\right), Y^{n}\left(\hat{t}_{n}\right)\right)=\left(\hat{x}_{n}, \hat{y}_{n}\right)$. Note that these processes are well defined on $\left[\hat{t}_{n}, \tau_{n}\right]$ where

$$
\tau_{n}:=\inf \left\{t \geq \hat{t}_{n}:\left(t, X^{n}(t)\right) \notin B_{r}\left(t_{0}, x_{0}\right) \text { or }\left|Y^{n}(t)-\varphi\left(t, X^{n}(t)\right)\right| \geq r\right\} .
$$

Applying Itô's Lemma to $Y^{n}-\varphi\left(\cdot, X^{n}\right)$ and arguing as in the proof of Lemma 3.4, we then deduce that (3.24) and (3.25) lead to a contradiction to (DP2) of Corollary 3.1.

\subsubsection{A comparison result}

We now prove Theorem 3.3. This is a consequence of the following comparison result and the growth property for $v$ which was derived in Remark 3.4.

Proposition 3.1 Let Assumption 3.2 hold. Let $V$ (resp. U) be a non-negative lower-semicontinuous (resp. upper-semicontinuous) locally bounded map on $[0, T] \times(0, \infty)^{d}$ satisfying (3.11). Assume that $V($ resp. $U)$ is a supersolution (resp. subsolution) of $(3.8)$ on $[0, T) \times(0, \infty)^{d}$ such that $V(T, \cdot)$ (resp. $U(T, \cdot))$ is a supersolution (resp. subsolution) of $(3.10)$ on $(0, \infty)^{d}$. Then, $V \geq U$ on $[0, T] \times(0, \infty)^{d}$.

Proof. 1. As usual, we first fix $\kappa>0$ and introduce the functions $\tilde{U}(t, x):=e^{\kappa t} U(t, x), \tilde{V}(t, x):=$ $e^{\kappa t} V(t, x)$ and $\tilde{g}(t, x):=e^{\kappa t} g(t, x)$, so that the function $\tilde{V}$ (resp. $\tilde{U}$ ) is a viscosity supersolution (resp. subsolution) of $\tilde{\mathcal{H}} \varphi=0$, where for a smooth function $\varphi$

$$
\tilde{\mathcal{H}} \varphi(t, x):=\min \left\{\sup _{\pi \in \mathcal{N} \varphi(t, x)}\left(\pi^{\prime} \mu(x) \varphi(t, x)+\kappa \varphi(t, x)-\mathcal{L} \varphi(t, x)\right), \varphi(t, x)-\tilde{g}(t, x)\right\} .
$$

Let $\lambda>1$ and $\bar{\gamma} \in(0, \infty)^{d}$ be as in Assumption 3.2. Let $\beta$ be defined by $\beta(t, x)=e^{\tau(T-t)}\left(1+x^{\lambda \bar{\gamma}}\right)$, for some $\tau>0$ to be chosen below, and observe that the fact that $\delta_{x} \geq 0$ and $\lambda \bar{\gamma} \in K_{x}$ for all $x \in(0, \infty)^{d}$ implies that

$$
\mathcal{M} \beta(t, x)=\inf _{\rho \in \tilde{K}_{x}}\left(\delta_{x}(\rho)+x^{\lambda \bar{\gamma}}\left[\delta_{x}(\rho)-\rho^{\prime}(\lambda \bar{\gamma})\right]\right) e^{\tau(T-t)} \geq 0 \quad \forall(t, x) \in[0, T] \times(0, \infty)^{d} .
$$

Moreover, one easily checks, by using the fact that $\mu$ and $\sigma$ are bounded, and $K$ is compact, that we can choose $\tau$ large enough so that, on $[0, T] \times(0, \infty)^{d}$,

$\min \left\{-2 L\left|D \beta(t, x)^{\prime} \operatorname{diag}[x] \mu(x)\right|+\kappa \beta(t, x)-\mathcal{L} \beta(t, x), \kappa \beta(t, x)-\partial_{t} \beta(t, x)-\frac{1}{2} \operatorname{Tr}\left[a(x) D^{2} \beta(t, x)\right]\right\} \geq 0$

where $a(z):=\operatorname{diag}[z] \sigma(z) \sigma(z)^{\prime} \operatorname{diag}[z]$, and $L$ is as in (v.a) of Assumption 3.2 if it holds and $L=0$ otherwise.

2. In order to show that $U \leq V$, we argue by contradiction. We therefore assume that

$$
\sup _{[0, T] \times(0, \infty)^{d}}(U-V)>0
$$

and work towards a contradiction. 
2.1. Using the growth condition on $\tilde{U}$ and $\tilde{V}$, and (3.29), we deduce that

$$
0<2 m:=\sup _{[0, T] \times(0, \infty)^{d}}(\tilde{U}-\tilde{V}-2 \alpha \beta)<\infty
$$

for $\alpha>0$ small enough. Fix $\varepsilon>0$ and let $f$ be defined on $(0, \infty)^{d}$ by

$$
f(x)=\sum_{i=1}^{d}\left(x^{i}\right)^{-2} .
$$

Arguing as in the proof of Proposition 6.9 in [5], see also below for similar arguments, we obtain that

$$
\Phi^{\varepsilon}:=\tilde{U}-\tilde{V}-2(\alpha \beta+\varepsilon f)
$$

admits a maximum $\left(t_{\varepsilon}, x_{\varepsilon}\right)$ on $[0, T] \times(0, \infty)^{d}$, which, for $\varepsilon>0$ small enough, satisfies

$$
\Phi^{\varepsilon}\left(t_{\varepsilon}, x_{\varepsilon}\right) \geq m>0
$$

as well as

$$
\limsup _{\varepsilon \rightarrow 0} \varepsilon\left(\left|f\left(x_{\varepsilon}\right)\right|+\left|\operatorname{diag}\left[x_{\varepsilon}\right] D f\left(x_{\varepsilon}\right)\right|+\left|\mathcal{L} f\left(x_{\varepsilon}\right)\right|\right)=0 .
$$

2.2. For $n \geq 1$ and $\zeta \in(0,1)$, we then define the function $\Psi_{n, \zeta}^{\varepsilon}$ on $[0, T] \times(0, \infty)^{2 d}$ by

$$
\Psi_{n, \zeta}^{\varepsilon}(t, x, y):=\Theta(t, x, y)-\varepsilon(f(x)+f(y))-\zeta\left(\left|x-x_{\varepsilon}\right|^{2}+\left|t-t_{\varepsilon}\right|^{2}\right)-n^{2}|x-y|^{2},
$$

where

$$
\Theta(t, x, y):=\tilde{U}(t, x)-\tilde{V}(t, y)-\alpha(\beta(t, x)+\beta(t, y))
$$

It follows from the growth condition on $\tilde{U}$ and $\tilde{V}$ that $\Psi_{n, \zeta}^{\varepsilon}$ attains its maximum at some $\left(t_{n}^{\varepsilon}, x_{n}^{\varepsilon}, y_{n}^{\varepsilon}\right)$ $\in[0, T] \times(0, \infty)^{2 d}$. Moreover, the inequality $\Psi_{n, \zeta}^{\varepsilon}\left(t_{n}^{\varepsilon}, x_{n}^{\varepsilon}, y_{n}^{\varepsilon}\right) \geq \Psi_{n, \zeta}^{\varepsilon}\left(t_{\varepsilon}, x_{\varepsilon}, x_{\varepsilon}\right)$ implies that

$$
\begin{aligned}
\Theta\left(t_{n}^{\varepsilon}, x_{n}^{\varepsilon}, y_{n}^{\varepsilon}\right) & \geq \Theta\left(t_{\varepsilon}, x_{\varepsilon}, x_{\varepsilon}\right)-2 \varepsilon f\left(x_{\varepsilon}\right) \\
& +n^{2}\left|x_{n}^{\varepsilon}-y_{n}^{\varepsilon}\right|^{2}+\zeta\left(\left|x_{n}^{\varepsilon}-x_{\varepsilon}\right|^{2}+\left|t_{n}^{\varepsilon}-t_{\varepsilon}\right|^{2}\right)+\varepsilon\left(f\left(x_{n}^{\varepsilon}\right)+f\left(y_{n}^{\varepsilon}\right)\right)
\end{aligned}
$$

Using the growth property of $\tilde{U}$ and $\tilde{V}$ again, we deduce that the term on the second line is bounded in $n$ so that, up to a subsequence,

$$
x_{n}^{\varepsilon}, y_{n}^{\varepsilon} \underset{n \rightarrow \infty}{\longrightarrow} \bar{x}^{\varepsilon} \in(0, \infty)^{d} \text { and } t_{n}^{\varepsilon} \underset{n \rightarrow \infty}{\longrightarrow} \bar{t}^{\varepsilon} \in[0, T] .
$$

Sending $n \rightarrow \infty$ in the previous inequality and using the maximum property of $\left(t_{\varepsilon}, x_{\varepsilon}\right)$, we also get

$$
\begin{aligned}
0 & \geq \Phi^{\varepsilon}\left(\bar{t}^{\varepsilon}, \bar{x}^{\varepsilon}\right)-\Phi^{\varepsilon}\left(t_{\varepsilon}, x_{\varepsilon}\right) \\
& \geq \limsup _{n \rightarrow \infty}\left(n^{2}\left|x_{n}^{\varepsilon}-y_{n}^{\varepsilon}\right|^{2}+\zeta\left(\left|x_{n}^{\varepsilon}-x_{\varepsilon}\right|^{2}+\left|t_{n}^{\varepsilon}-t_{\varepsilon}\right|^{2}\right)\right)
\end{aligned}
$$

which shows that

$$
\begin{aligned}
& \text { (a) } n^{2}\left|x_{n}^{\varepsilon}-y_{n}^{\varepsilon}\right|^{2}+\zeta\left(\left|x_{n}^{\varepsilon}-x_{\varepsilon}\right|^{2}+\left|t_{n}^{\varepsilon}-t_{\varepsilon}\right|^{2}\right) \underset{n \rightarrow \infty}{\longrightarrow} 0, \\
& \text { (b) } \tilde{U}\left(t_{n}^{\varepsilon}, x_{n}^{\varepsilon}\right)-\tilde{V}\left(t_{n}^{\varepsilon}, y_{n}^{\varepsilon}\right) \underset{n \rightarrow \infty}{\longrightarrow}(\tilde{U}-\tilde{V})\left(t_{\varepsilon}, x_{\varepsilon}\right) \geq m+2 \alpha \beta\left(t_{\varepsilon}, x_{\varepsilon}\right)+2 \varepsilon f\left(x_{\varepsilon}\right)>0,
\end{aligned}
$$

where we used (3.32) for the last assertion. 
3.1. Assume that, after possibly passing to a subsequence, $t_{n}^{\varepsilon}=T$, for all $n \geq 1$. Then, Ishii's Lemma, see e.g. [1], and the viscosity property of $U(T, \cdot)$ and $V(T, \cdot)$ imply that

$$
\begin{aligned}
& \min \left\{M\left(x_{n}^{\varepsilon}, \tilde{U}\left(T, x_{n}^{\varepsilon}\right), p_{n}^{\varepsilon}\right), \tilde{U}\left(T, x_{n}^{\varepsilon}\right)-\tilde{g}\left(T, x_{n}^{\varepsilon}\right)\right\} \leq 0 \\
& \min \left\{M\left(y_{n}^{\varepsilon}, \tilde{V}\left(T, y_{n}^{\varepsilon}\right), q_{n}^{\varepsilon}\right), \tilde{V}\left(T, y_{n}^{\varepsilon}\right)-\tilde{g}\left(T, y_{n}^{\varepsilon}\right)\right\} \geq 0,
\end{aligned}
$$

where

$$
\begin{aligned}
p_{n}^{\varepsilon} & :=2 n^{2}\left(x_{n}^{\varepsilon}-y_{n}^{\varepsilon}\right)+2 \zeta\left(x_{n}^{\varepsilon}-x_{\varepsilon}\right)+\alpha D \beta\left(t_{n}^{\varepsilon}, x_{n}^{\varepsilon}\right)+\varepsilon D f\left(x_{n}^{\varepsilon}\right) \\
q_{n}^{\varepsilon}: & :=2 n^{2}\left(x_{n}^{\varepsilon}-y_{n}^{\varepsilon}\right)-\alpha D \beta\left(t_{n}^{\varepsilon}, y_{n}^{\varepsilon}\right)-\varepsilon D f\left(y_{n}^{\varepsilon}\right) .
\end{aligned}
$$

Assuming that, after possibly passing to a subsequence, $\tilde{U}\left(T, x_{n}^{\varepsilon}\right) \leq \tilde{g}\left(T, x_{n}^{\varepsilon}\right)$ for all $n \geq 1$, we get a contradiction to (b) since $\tilde{V}\left(T, y_{n}^{\varepsilon}\right) \geq \tilde{g}\left(T, y_{n}^{\varepsilon}\right)$ so that passing to the limit, recall (a) and the fact that $g$ is continuous, implies $\tilde{U}\left(T, x_{\varepsilon}\right) \leq \tilde{g}\left(T, x_{\varepsilon}\right) \leq \tilde{V}\left(T, x_{\varepsilon}\right)$.

We can therefore assume that $\tilde{U}\left(T, x_{n}^{\varepsilon}\right)>\tilde{g}\left(T, x_{n}^{\varepsilon}\right)$ for all $n$. Using the two above inequalities, we then deduce that

$$
0 \geq M\left(x_{n}^{\varepsilon}, \tilde{U}\left(T, x_{n}^{\varepsilon}\right), p_{n}^{\varepsilon}\right)-M\left(y_{n}^{\varepsilon}, \tilde{V}\left(T, y_{n}^{\varepsilon}\right), q_{n}^{\varepsilon}\right)
$$

which implies that we can find $\hat{\rho}_{x_{n}^{\varepsilon}} \in \tilde{K}_{x_{n}^{\varepsilon}}$ such that for all $\rho_{n}^{\varepsilon} \in \tilde{K}_{y_{n}^{\varepsilon}}$ we have

$$
\begin{aligned}
0 & \geq \delta_{x_{n}^{\varepsilon}}\left(\hat{\rho}_{x_{n}^{\varepsilon}}\right)\left[\Theta\left(t_{n}^{\varepsilon}, x_{n}^{\varepsilon}, y_{n}^{\varepsilon}\right)-\varepsilon\left(f\left(x_{n}^{\varepsilon}\right)+f\left(y_{n}^{\varepsilon}\right)\right]+\left(\delta_{x_{n}^{\varepsilon}}\left(\hat{\rho}_{x_{n}^{\varepsilon}}\right)-\delta_{y_{n}^{\varepsilon}}\left(\rho_{n}^{\varepsilon}\right)\right)\left(\tilde{V}\left(T, y_{n}^{\varepsilon}\right)+\alpha \beta\left(y_{n}^{\varepsilon}\right)+\varepsilon f\left(y_{n}^{\varepsilon}\right)\right)\right. \\
& +\alpha M\left(x_{n}^{\varepsilon}, \beta\left(x_{n}^{\varepsilon}\right), D \beta\left(t_{n}^{\varepsilon}, x_{n}^{\varepsilon}\right)\right)+\varepsilon M\left(x_{n}^{\varepsilon}, f\left(x_{n}^{\varepsilon}\right), f\left(x_{n}^{\varepsilon}\right)\right) \\
& +\alpha M\left(y_{n}^{\varepsilon}, \beta\left(y_{n}^{\varepsilon}\right), D \beta\left(t_{n}^{\varepsilon}, y_{n}^{\varepsilon}\right)\right)+\varepsilon M\left(y_{n}^{\varepsilon}, f\left(y_{n}^{\varepsilon}\right), f\left(y_{n}^{\varepsilon}\right)\right) \\
& -2 \zeta\left(\hat{\rho}_{x_{n}^{\varepsilon}}\right)^{\prime} \operatorname{diag}\left[x_{n}^{\varepsilon}\right]\left(x_{n}^{\varepsilon}-x_{\varepsilon}\right)-2 n^{2}\left(\left(\hat{\rho}_{x_{n}^{\varepsilon}}\right)^{\prime} \operatorname{diag}\left[x_{n}^{\varepsilon}\right]-\left(\rho_{n}^{\varepsilon}\right)^{\prime} \operatorname{diag}\left[y_{n}^{\varepsilon}\right]\right)\left(x_{n}^{\varepsilon}-y_{n}^{\varepsilon}\right)
\end{aligned}
$$

In view of (iv) of Assumption 3.2 and (a) above, we can choose $\rho_{n}^{\varepsilon}$ such that, for some $C>0$,

$$
\left|\hat{\rho}_{x_{n}^{\varepsilon}}-\rho_{n}^{\varepsilon}\right| \leq C\left|x_{n}^{\varepsilon}-y_{n}^{\varepsilon}\right| \quad \text { and } \quad \delta_{x_{n}^{\varepsilon}}\left(\hat{\rho}_{x_{n}^{\varepsilon}}\right)-\delta_{y_{n}^{\varepsilon}}\left(\rho_{n}^{\varepsilon}\right) \geq-\epsilon\left(x_{n}^{\varepsilon}, y_{n}^{\varepsilon}\right) \rightarrow 0 .
$$

Using (a), (b), (iii) of Assumption 3.2, the fact that $\tilde{V}, \beta, f \geq 0,(3.27)$ and (3.33), the previous inequality applied to $\varepsilon>0$ small enough and $n$ large enough leads to

$$
0 \geq c_{K}(m / 2)
$$

which contradicts (3.32).

3.2. In view of the above point, we can now assume, after possibly passing to a subsequence, that $t_{n}^{\varepsilon}<T$ for all $n \geq 1$. From Ishii's Lemma, see Theorem 8.3 in [9], we deduce that, for each $\eta>0$, there are real coefficients $b_{1, n}^{\varepsilon}, b_{2, n}^{\varepsilon}$ and symmetric matrices $\mathcal{X}_{n}^{\varepsilon, \eta}$ and $\mathcal{Y}_{n}^{\varepsilon, \eta}$ such that

$$
\left(b_{1, n}^{\varepsilon}, p_{n}^{\varepsilon}, \mathcal{X}_{n}^{\varepsilon, \eta}\right) \in \overline{\mathcal{P}}_{\overline{\mathcal{O}}}^{+} \tilde{U}\left(t_{n}^{\varepsilon}, x_{n}^{\varepsilon}\right) \quad \text { and } \quad\left(-b_{2, n}^{\varepsilon}, q_{n}^{\varepsilon}, \mathcal{Y}_{n}^{\varepsilon, \eta}\right) \in \overline{\mathcal{P}}_{\overline{\mathcal{O}}}^{-} \tilde{V}\left(t_{n}^{\varepsilon}, y_{n}^{\varepsilon}\right)
$$

see [9] for the standard notations $\overline{\mathcal{P}}_{\overline{\mathcal{O}}}^{+}$and $\overline{\mathcal{P}}_{\overline{\mathcal{O}}}^{-}$, where

$$
\begin{aligned}
p_{n}^{\varepsilon} & :=2 n^{2}\left(x_{n}^{\varepsilon}-y_{n}^{\varepsilon}\right)+2 \zeta\left(x_{n}^{\varepsilon}-x_{\varepsilon}\right)+\alpha D \beta\left(t_{n}^{\varepsilon}, x_{n}^{\varepsilon}\right)+\varepsilon D f\left(x_{n}^{\varepsilon}\right) \\
q_{n}^{\varepsilon} & :=2 n^{2}\left(x_{n}^{\varepsilon}-y_{n}^{\varepsilon}\right)-\alpha D \beta\left(t_{n}^{\varepsilon}, y_{n}^{\varepsilon}\right)-\varepsilon D f\left(y_{n}^{\varepsilon}\right),
\end{aligned}
$$

and $b_{1, n}^{\varepsilon}, b_{2, n}^{\varepsilon}, \mathcal{X}_{n}^{\varepsilon, \eta}$ and $\mathcal{Y}_{n}^{\varepsilon, \eta}$ satisfy

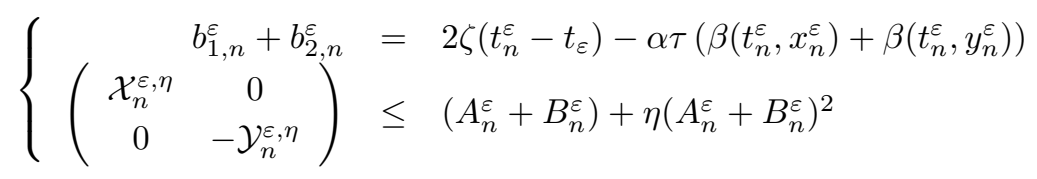


with

$A_{n}^{\varepsilon}:=\left(\begin{array}{cc}2 n^{2} I_{d}+2 \zeta I_{d} & -2 n^{2} I_{d} \\ -2 n^{2} I_{d} & 2 n^{2} I_{d}\end{array}\right), B_{n}^{\varepsilon}:=\left(\begin{array}{cc}\alpha D^{2} \beta\left(t_{n}^{\varepsilon}, x_{n}^{\varepsilon}\right)+\varepsilon D^{2} f\left(x_{n}^{\varepsilon}\right) & 0 \\ 0 & \alpha D^{2} \beta\left(t_{n}^{\varepsilon}, y_{n}^{\varepsilon}\right)+\varepsilon D^{2} f\left(y_{n}^{\varepsilon}\right)\end{array}\right)$,

and $I_{d}$ stands for the $d \times d$ identity matrix.

3.2.a. Assume that, after possibly passing to a subsequence, either $N\left(x_{n}^{\varepsilon}, \tilde{U}\left(t_{n}^{\varepsilon}, x_{n}^{\varepsilon}\right), p_{n}^{\varepsilon}\right)=\emptyset$ or $M\left(x_{n}^{\varepsilon}, \tilde{U}\left(t_{n}^{\varepsilon}, x_{n}^{\varepsilon}\right), p_{n}^{\varepsilon}\right) \leq 0$. It then follows from Lemma 3.2 that, in both cases, $M\left(x_{n}^{\varepsilon}, \tilde{U}\left(t_{n}^{\varepsilon}, x_{n}^{\varepsilon}\right), p_{n}^{\varepsilon}\right) \leq$ 0 . Since the supersolution property of $\tilde{V}$ ensures that $M\left(y_{n}^{\varepsilon}, \tilde{V}\left(t_{n}^{\varepsilon}, y_{n}^{\varepsilon}\right), q_{n}^{\varepsilon}\right) \geq 0$, arguing as in Step 2.2. above leads to a contradiction. Similarly, we can not have $U\left(t_{n}^{\varepsilon}, x_{n}^{\varepsilon}\right) \leq g\left(t_{n}^{\varepsilon}, y_{n}^{\varepsilon}\right)$ along a subsequence since $V \geq g$ and $g$ is continuous, recall (a) and (b).

We can then assume that $N\left(x_{n}^{\varepsilon}, \tilde{U}\left(t_{n}^{\varepsilon}, x_{n}^{\varepsilon}\right), p_{n}^{\varepsilon}\right) \neq \emptyset, M\left(x_{n}^{\varepsilon}, \tilde{U}\left(t_{n}^{\varepsilon}, x_{n}^{\varepsilon}\right), p_{n}^{\varepsilon}\right)>0$ and $U\left(t_{n}^{\varepsilon}, x_{n}^{\varepsilon}\right)>g\left(t_{n}^{\varepsilon}, x_{n}^{\varepsilon}\right)$ for all $n \geq 1$, after possibly passing to a subsequence. It then follows from the super- and subsolution properties of $\tilde{V}$ and $\tilde{U}$, see Step 1., the fact that $K$ is compact and Lemma 3.2 that there exists $\pi\left(x_{n}^{\varepsilon}\right), \pi\left(y_{n}^{\varepsilon}\right) \in K$ such that

$$
\pi\left(x_{n}^{\varepsilon}\right) \in N\left(x_{n}^{\varepsilon}, \tilde{U}\left(t_{n}^{\varepsilon}, x_{n}^{\varepsilon}\right), p_{n}^{\varepsilon}\right) \text { and } \pi\left(y_{n}^{\varepsilon}\right) \in N\left(y_{n}^{\varepsilon}, \tilde{V}\left(t_{n}^{\varepsilon}, y_{n}^{\varepsilon}\right), q_{n}^{\varepsilon}\right)
$$

and, recall (3.34),

$$
\begin{aligned}
\kappa\left(\tilde{U}\left(t_{n}^{\varepsilon}, x_{n}^{\varepsilon}\right)-\tilde{V}\left(t_{n}^{\varepsilon}, y_{n}^{\varepsilon}\right)\right) & \leq-\tilde{U}\left(t_{n}^{\varepsilon}, x_{n}^{\varepsilon}\right) \pi\left(x_{n}^{\varepsilon}\right)^{\prime} \mu\left(x_{n}^{\varepsilon}\right)+\tilde{V}\left(t_{n}^{\varepsilon}, y_{n}^{\varepsilon}\right) \pi\left(y_{n}^{\varepsilon}\right)^{\prime} \mu\left(y_{n}^{\varepsilon}\right)+b_{1, n}^{\varepsilon}+b_{2, n}^{\varepsilon} \\
& +\bar{\mu}\left(x_{n}^{\varepsilon}\right)^{\prime} p_{n}^{\varepsilon}-\bar{\mu}\left(y_{n}^{\varepsilon}\right)^{\prime} q_{n}^{\varepsilon}+\frac{1}{2} \operatorname{Tr}\left[a\left(x_{n}^{\varepsilon}\right) \mathcal{X}_{n}^{\varepsilon, \eta}-a\left(y_{n}^{\varepsilon}\right) \mathcal{Y}_{n}^{\varepsilon, \eta}\right] \\
& \leq-\tilde{U}\left(t_{n}^{\varepsilon}, x_{n}^{\varepsilon}\right) \pi\left(x_{n}^{\varepsilon}\right)^{\prime} \mu\left(x_{n}^{\varepsilon}\right)+\tilde{V}\left(t_{n}^{\varepsilon}, y_{n}^{\varepsilon}\right) \pi\left(y_{n}^{\varepsilon}\right)^{\prime} \mu\left(y_{n}^{\varepsilon}\right) \\
& +2 \zeta\left(t_{n}^{\varepsilon}-x_{\varepsilon}\right)-\alpha \tau\left(\beta\left(t_{n}^{\varepsilon}, x_{n}^{\varepsilon}\right)+\beta\left(t_{n}^{\varepsilon}, y_{n}^{\varepsilon}\right)\right) \\
& +\bar{\mu}\left(x_{n}^{\varepsilon}\right)^{\prime} p_{n}^{\varepsilon}-\bar{\mu}\left(y_{n}^{\varepsilon}\right)^{\prime} q_{n}^{\varepsilon}+\frac{1}{2} \operatorname{Tr}\left[\Xi\left(t_{n}^{\varepsilon}, x_{n}^{\varepsilon}, y_{n}^{\varepsilon}\right)\left(A_{n}^{\varepsilon}+B_{n}^{\varepsilon}+\eta\left(A_{n}^{\varepsilon}+B_{n}^{\varepsilon}\right)^{2}\right)\right]
\end{aligned}
$$

where $\bar{\sigma}(z):=\operatorname{diag}[z] \sigma(z), \bar{\mu}(z)=\operatorname{diag}[z] \mu(z)$ and the positive semi-definite matrix $\Xi\left(t_{n}^{\varepsilon}, x_{n}^{\varepsilon}, y_{n}^{\varepsilon}\right)$ is defined by

$$
\Xi\left(x_{n}^{\varepsilon}, y_{n}^{\varepsilon}\right):=\left(\begin{array}{cc}
\bar{\sigma}\left(x_{n}^{\varepsilon}\right) \bar{\sigma}^{\prime}\left(x_{n}^{\varepsilon}\right) & \bar{\sigma}\left(y_{n}^{\varepsilon}\right) \bar{\sigma}^{\prime}\left(x_{n}^{\varepsilon}\right) \\
\bar{\sigma}\left(x_{n}^{\varepsilon}\right) \bar{\sigma}^{\prime}\left(y_{n}^{\varepsilon}\right) & \bar{\sigma}\left(y_{n}^{\varepsilon}\right) \bar{\sigma}^{\prime}\left(y_{n}^{\varepsilon}\right)
\end{array}\right) .
$$

3.2.b. We now assume that (v.a) of Assumption 3.2 holds. Then, for $n$ large enough, we can choose $\pi\left(x_{n}^{\varepsilon}\right)$ such that

$$
\left|\tilde{V}\left(t_{n}^{\varepsilon}, y_{n}^{\varepsilon}\right) \pi\left(y_{n}^{\varepsilon}\right)^{\prime} \mu\left(y_{n}^{\varepsilon}\right)-\tilde{U}\left(t_{n}^{\varepsilon}, x_{n}^{\varepsilon}\right) \pi\left(x_{n}^{\varepsilon}\right)^{\prime} \mu\left(x_{n}^{\varepsilon}\right)\right| \leq L\left|\left(q_{n}^{\varepsilon}\right)^{\prime} \operatorname{diag}\left[y_{n}^{\varepsilon}\right] \mu\left(y_{n}^{\varepsilon}\right)-\left(p_{n}^{\varepsilon}\right)^{\prime} \operatorname{diag}\left[x_{n}^{\varepsilon}\right] \mu\left(x_{n}^{\varepsilon}\right)\right| .
$$

It then follows from (3.35) and (3.36) that

$$
\begin{aligned}
\kappa\left(\tilde{U}\left(t_{n}^{\varepsilon}, x_{n}^{\varepsilon}\right)-\tilde{V}\left(t_{n}^{\varepsilon}, y_{n}^{\varepsilon}\right)\right) & \leq L\left|\left(q_{n}^{\varepsilon}\right)^{\prime} \operatorname{diag}\left[y_{n}^{\varepsilon}\right] \mu\left(y_{n}^{\varepsilon}\right)-\left(p_{n}^{\varepsilon}\right)^{\prime} \operatorname{diag}\left[x_{n}^{\varepsilon}\right] \mu\left(x_{n}^{\varepsilon}\right)\right| \\
& +2 \zeta\left(t_{n}^{\varepsilon}-x_{\varepsilon}\right)-\alpha \tau\left(\beta\left(t_{n}^{\varepsilon}, x_{n}^{\varepsilon}\right)+\beta\left(t_{n}^{\varepsilon}, y_{n}^{\varepsilon}\right)\right) \\
& +\bar{\mu}\left(x_{n}^{\varepsilon}\right)^{\prime} p_{n}^{\varepsilon}-\bar{\mu}\left(y_{n}^{\varepsilon}\right)^{\prime} q_{n}^{\varepsilon}+\frac{1}{2} \operatorname{Tr}\left[\Xi\left(x_{n}^{\varepsilon}, y_{n}^{\varepsilon}\right)\left(A_{n}^{\varepsilon}+B_{n}^{\varepsilon}+\eta\left(A_{n}^{\varepsilon}+B_{n}^{\varepsilon}\right)^{2}\right)\right] .
\end{aligned}
$$

Using (a)-(b), (3.28) and (3.33), we deduce that we can find $C>0$ independent of $(\eta, \zeta)$ such that for $\varepsilon$ small and $n$ large enough

$$
\begin{aligned}
\kappa \frac{m}{2} & \leq \kappa\left(\tilde{U}\left(t_{n}^{\varepsilon}, x_{n}^{\varepsilon}\right)-\tilde{V}\left(t_{n}^{\varepsilon}, y_{n}^{\varepsilon}\right)-(\alpha \beta+\varepsilon f)\left(t_{n}^{\varepsilon}, x_{n}^{\varepsilon}\right)-(\alpha \beta+\varepsilon f)\left(t_{n}^{\varepsilon}, y_{n}^{\varepsilon}\right)\right) \\
& \leq C(1+\zeta) \theta(\varepsilon, n)+\frac{1}{2} \operatorname{Tr}\left[\Xi\left(x_{n}^{\varepsilon}, y_{n}^{\varepsilon}\right)\left(A_{n}^{\varepsilon}+\eta\left(A_{n}^{\varepsilon}+B_{n}^{\varepsilon}\right)^{2}\right)\right]
\end{aligned}
$$


where $\theta(\varepsilon, n)$ is independent of $(\eta, \zeta)$ and satisfies

$$
\limsup _{\varepsilon \rightarrow 0} \limsup _{n \rightarrow \infty}|\theta(\varepsilon, n)|=0 .
$$

Sending $\eta \rightarrow 0$ in the previous inequality provides

$$
\kappa \frac{m}{2} \leq C(1+\zeta) \theta(\varepsilon, n)+\frac{1}{2} \operatorname{Tr}\left[\Xi\left(x_{n}^{\varepsilon}, y_{n}^{\varepsilon}\right) A_{n}^{\varepsilon}\right],
$$

so that

$$
\kappa \frac{m}{2} \leq C(1+\zeta) \theta(\varepsilon, n)+\zeta \operatorname{Tr}\left[\bar{\sigma}\left(x_{n}^{\varepsilon}\right) \bar{\sigma}^{\prime}\left(x_{n}^{\varepsilon}\right)\right]+n^{2}\left|\operatorname{diag}\left[x_{n}^{\varepsilon}\right] \sigma\left(x_{n}^{\varepsilon}\right)-\operatorname{diag}\left[y_{n}^{\varepsilon}\right] \sigma\left(y_{n}^{\varepsilon}\right)\right|^{2} .
$$

Finally, using (a) and the Lipschitz continuity of the coefficients, we obtain by sending $n$ to $\infty$ and then $\zeta$ to 0 in the last inequality that $\kappa m \leq 0$, which is the required contradiction and concludes the proof.

3.2.c. In the case where (v.b) of Assumption 3.2 holds. Then, (3.35) and (3.36) imply that

$$
\begin{aligned}
\kappa\left(\tilde{U}\left(t_{n}^{\varepsilon}, x_{n}^{\varepsilon}\right)-\tilde{V}\left(t_{n}^{\varepsilon}, y_{n}^{\varepsilon}\right)\right) & \leq 2 \zeta\left(t_{n}^{\varepsilon}-x_{\varepsilon}\right)-\alpha \tau\left(\beta\left(t_{n}^{\varepsilon}, x_{n}^{\varepsilon}\right)+\beta\left(t_{n}^{\varepsilon}, y_{n}^{\varepsilon}\right)\right) \\
& +\frac{1}{2} \operatorname{Tr}\left[\Xi\left(t_{n}^{\varepsilon}, x_{n}^{\varepsilon}, y_{n}^{\varepsilon}\right)\left(A_{n}^{\varepsilon}+B_{n}^{\varepsilon}+\eta\left(A_{n}^{\varepsilon}+B_{n}^{\varepsilon}\right)^{2}\right)\right]
\end{aligned}
$$

and the proof is concluded as in 3.2.b. above by using the fact that the right hand-side in the min in of (3.28) is non-negative (instead of the left hand-side as above).

\subsubsection{Proof of the "face-lifted" representation}

In this Section, we prove Corollary 3.2 and Corollary 3.3. We start with some preliminary results.

Lemma 3.6 Let (i)-(iv) of Assumption 3.2 hold. Fix $t_{0} \in[0, T]$ and let $V$ (resp. $U$ ) be a lowersemicontinuous (resp. upper-semicontinuous) viscosity supersolution (resp. subsolution) on $(0, \infty)^{d}$ of

$$
\min \left\{\mathcal{M} \phi, \phi-g\left(t_{0}, \cdot\right)\right\}=0
$$

Assume that $U$ and $V$ are non-negative and satisfy the growth condition (3.11). Then, $U \leq V$ on $(0, \infty)^{d}$.

Proof. This follows from the same line of arguments as in Steps 1., 2. and 3.1. of the proof of Proposition 3.1.

Lemma 3.7 Let (i)-(iv) of Assumption 3.2 hold. Then,

(i) There exists $C>0$ such that

$$
|\check{g}(t, x)| \leq C\left(1+x^{\bar{\gamma}}\right) \quad \text { for all }(t, x) \in[0, T] \times(0, \infty)^{d} .
$$

(ii) Assume further that the assumptions of Corollary 3.2 hold. Then, for each $t_{0} \in[0, T], \check{g}\left(t_{0}, \cdot\right)$ is continuous on $(0, \infty)^{d}$ and is the unique viscosity solution of (3.39) satisfying the growth condition (3.40).

Proof. 1. Fix $\rho \in L_{1}^{0}(L e b)$ and $\tau \geq 0$. It follows from Assumption 3.2 that we can find $C>0$ such that

$$
e^{-\int_{0}^{\tau} \delta_{\chi_{x}^{\rho}(s)}\left(\rho_{s}\right) d s} g\left(t_{0}, \chi_{x}^{\rho}(\tau)\right) \leq C\left(1+\prod_{i=1}^{d}\left(x^{i}\right)^{\bar{\gamma}^{i}} e^{\int_{0}^{\tau} \bar{\gamma}^{i} \bar{\rho}_{s}^{i} d s}\right) e^{-\int_{0}^{\tau} \delta_{\chi_{x}^{\rho}(s)}\left(\rho_{s}\right) d s} \leq C\left(1+x^{\bar{\gamma}}\right)
$$


where we used the fact $\delta_{\chi_{x}^{\rho}} \geq 0$ since $0 \in K_{z}$ for all $z \in(0, \infty)^{d}$, and the fact that $\bar{\gamma}^{\prime} \rho-\delta_{\chi_{x}^{\rho}}(\rho) \leq 0$ since $\bar{\gamma} \in K \subset K_{\chi_{x}^{\rho}}$.

2. We now prove that $\check{g}\left(t_{0}, \cdot\right)$ is a (discontinuous) viscosity solution of $(3.39)$. Let $\check{g}_{*}\left(t_{0}, \cdot\right)\left(\right.$ resp. $\left.\check{g}^{*}\left(t_{0}, \cdot\right)\right)$ be the lower-semicontinuous (resp. upper-semicontinuous) envelope of $x \mapsto \check{g}\left(t_{0}, x\right)$.

Note that $\check{g}\left(t_{0}, \cdot\right)$ satisfies the dynamic programming principle

$$
\check{g}\left(t_{0}, x\right)=\sup _{(\rho, \tau) \in L_{1}^{0}(L e b) \times \mathbb{R}_{+}} e^{-\int_{0}^{\tau \wedge h} \delta_{\chi_{x}^{\rho}(s)}\left(\rho_{s}\right) d s}\left(g\left(t_{0}, \chi_{x}^{\rho}(\tau)\right) \mathbf{1}_{\tau \leq h}+\check{g}\left(t_{0}, \chi_{x}^{\rho}(h)\right) \mathbf{1}_{\tau>h}\right), h>0 .
$$

2.a. We start with the supersolution property. Note that $\check{g} \geq g$ by construction (take $\rho=0$ and $\tau=0$ in the definition of $\check{g}$ or in (3.41)). Let $\phi$ be a non-negative smooth function and let $x_{0} \in(0, \infty)^{d}$ be a strict minimum point of $\check{g}_{*}\left(t_{0}, \cdot\right)-\phi$ such that $\check{g}_{*}\left(t_{0}, x_{0}\right)-\phi\left(x_{0}\right)=0$. Let $\left(x_{n}\right)_{n}$ be a sequence in $(0, \infty)^{d}$ such that $\check{g}\left(t_{0}, x_{n}\right) \rightarrow \check{g}_{*}\left(t_{0}, x_{0}\right)$. Assume that

$$
\inf _{\rho \in \tilde{K}_{x_{0}}}\left(\delta_{x_{0}}(\rho) \phi\left(x_{0}\right)-\rho^{\prime} \operatorname{diag}\left[x_{0}\right] D \phi\left(x_{0}\right)\right)<0 .
$$

It then follows from (iv) of Assumption 3.2 that we can find $r, m>0$ and a Lipschitz continuous map $\hat{\rho}$ such that, for some $\eta>0$,

$$
\delta_{x}(\hat{\rho}(x)) \leq \eta \quad \text { and } \quad \delta_{x}(\hat{\rho}(x)) \phi(x)-\hat{\rho}(x)^{\prime} \operatorname{diag}[x] D \phi(x)<-m \forall x \in B_{r}\left(x_{0}\right) .
$$

This implies that $\hat{\chi}_{n}$ defined as $\chi_{x_{n}}^{\rho}$ for the Markovian control $\rho=\hat{\rho}\left(\hat{\chi}_{n}\right)$ satisfies

$$
\phi\left(x_{n}\right)<e^{-\int_{0}^{h_{n}} \delta_{\hat{\chi}_{n}(s)}\left(\hat{\rho}\left(\hat{\chi}_{n}(s)\right)\right) d s}\left(\phi\left(\hat{\chi}_{n}\left(h_{n}\right)\right)-m h_{n}\right)
$$

where

$$
h_{n}:=\inf \left\{s \geq 0: \hat{\chi}_{n}(s) \notin B_{r}\left(x_{0}\right)\right\} \wedge h
$$

for some $h>0$. Since $x_{0}$ is a strict minimum point of $\check{g}_{*}\left(t_{0}, \cdot\right)-\phi$, we can then find $\zeta>0$ such that

$$
\phi\left(x_{n}\right)<e^{-\int_{0}^{h_{n}} \delta_{\hat{\chi}_{n}(s)}\left(\hat{\rho}\left(\hat{\chi}_{n}(s)\right)\right) d s}\left(-\zeta \mathbf{1}_{h_{n}<h}+\check{g}\left(t_{0}, \hat{\chi}_{n}\left(h_{n}\right)\right)-m h_{n}\right) .
$$

Using the left-hand side of (3.42), we then obtain

$$
\phi\left(x_{n}\right)<e^{-\int_{0}^{h_{n}} \delta_{\hat{\chi}_{n}(s)}\left(\hat{\rho}\left(\hat{\chi}_{n}(s)\right)\right) d s} \check{g}\left(t_{0}, \hat{\chi}_{n}\left(h_{n}\right)\right)-e^{-h \eta}\left(\zeta \mathbf{1}_{h_{n}<h}+m h \mathbf{1}_{h_{n}=h}\right) .
$$

Since $\check{g}\left(t_{0}, x_{n}\right)-\phi\left(x_{n}\right) \rightarrow 0$, this leads to a contradiction to (3.41) for $n$ large enough.

2.b. We now turn to the subsolution property. Let $\phi$ be a non-negative smooth function and let $x_{0} \in(0, \infty)^{d}$ be a strict maximum point of $\check{g}^{*}\left(t_{0}, \cdot\right)-\phi$ such that $\check{g}^{*}\left(t_{0}, x_{0}\right)-\phi\left(x_{0}\right)=0$. Let $\left(x_{n}\right)_{n}$ be a sequence in $(0, \infty)^{d}$ such that $\check{g}\left(t_{0}, x_{n}\right) \rightarrow \check{g}^{*}\left(t_{0}, x_{0}\right)$. Assume that

$$
\min \left\{\inf _{\rho \in \tilde{K}_{x_{0}}}\left(\delta_{x_{0}}(\rho) \phi\left(x_{0}\right)-\rho^{\prime} \operatorname{diag}\left[x_{0}\right] D \phi\left(x_{0}\right)\right), \phi\left(x_{0}\right)-g\left(t_{0}, x_{0}\right)\right\}>0 .
$$

Since $g \geq 0$, this implies that $\phi>0$ on $B_{r}\left(x_{0}\right)$, for some $r>0$. Moreover, the fact that $\tilde{K}_{x_{0}}$ is compact implies that, after possibly changing $r>0$, we can find $\varepsilon>0$ such that $\phi(x)-\varepsilon>0$ on $B_{r}\left(x_{0}\right)$ and $M\left(x_{0}, \phi\left(x_{0}\right)-\varepsilon, D \phi\left(x_{0}\right)\right)>0$. In view of Lemma 3.2, this implies that $\left(\phi\left(x_{0}\right)-\varepsilon\right)^{-1} \operatorname{diag}\left[x_{0}\right] D \phi\left(x_{0}\right) \in$ $\operatorname{int}\left(K_{x_{0}}\right)$. It the follows from Assumption 3.1 that, after possibly changing $r>0, N(x, \phi(x)-\varepsilon, D \phi(x)) \neq$ $\emptyset$ on $B_{r}\left(x_{0}\right)$, which, by Lemma 3.2 again, implies that $M(x, \phi(x)-\varepsilon, D \phi(x)) \geq 0$ on $B_{r}\left(x_{0}\right)$. Since $\delta_{x} \geq c_{K}>0$, recall (iii) of Assumption 3.2, we deduce that $M(x, \phi(x), D \phi(x)) \geq \varepsilon c_{K}$ on $B_{r}\left(x_{0}\right)$. Using Lemma 3.2, the continuity of $g$ and (3.43), we finally obtain

$$
\min \left\{\inf _{\rho \in \tilde{K}_{x}}\left(\delta_{x}(\rho) \phi(x)-\rho^{\prime} \operatorname{diag}[x] D \phi(x)\right), \phi(x)-g\left(t_{0}, x\right)\right\} \geq m \quad \text { on } B_{r}\left(x_{0}\right)
$$


for some $m>0$. Moreover, it follows from Assumption (ii) of Corollary 3.2 that

$$
\sup \left\{\delta_{x}(\rho), \rho \in \tilde{K}_{x}\right\} \leq \eta \quad \text { on } B_{r}\left(x_{0}\right)
$$

for some $\eta>0$.

We now consider a sequence $\left(x_{n}\right)_{n}$ in $(0, \infty)^{d}$ such that $\check{g}\left(t_{0}, x_{n}\right) \rightarrow \check{g}^{*}\left(t_{0}, x_{0}\right)$. Given $\rho \in L_{1}^{0}(L e b)$, we set $h_{n}:=\inf \left\{s \geq 0: \chi_{x_{n}}^{\rho}(s) \notin B_{r}\left(x_{0}\right)\right\} \wedge h$ for some $h>0$. Then, (3.44) and the fact that $x_{0}$ is a strict maximum point of $\check{g}^{*}\left(t_{0}, \cdot\right)-\phi$ implies that we can find $\zeta>0$ such that, for all $\tau \geq 0$,

$$
\begin{aligned}
\phi\left(x_{n}\right) & \geq e^{-\int_{0}^{h_{n} \wedge \tau} \delta_{\chi_{x_{n}}^{\rho}(s)}\left(\rho_{s}\right) d s}\left(\phi\left(\chi_{x_{n}}^{\rho}\left(h_{n} \wedge \tau\right)\right)+m\left(h_{n} \wedge \tau\right)\right) \\
& \geq e^{-\int_{0}^{h_{n} \wedge \tau} \delta_{\chi_{x_{n}}^{\rho}(s)}\left(\rho_{s}\right) d s}\left(\check{g}\left(t_{0}, \chi_{x_{n}}^{\rho}\left(h_{n}\right)\right) \mathbf{1}_{h_{n}>\tau}+g\left(t_{0}, \chi_{x_{n}}^{\rho}(\tau)\right) \mathbf{1}_{\tau \leq h_{n}}+\zeta \wedge m \wedge(m h)\right) .
\end{aligned}
$$

Since $\phi\left(x_{n}\right)-\check{g}\left(t_{0}, x_{n}\right) \rightarrow 0$, the above inequality combined with (3.45) leads to a contradiction to (3.41) for $n$ large enough, by arbitrariness of $\tau$ and $\rho$.

We can now conclude the proof of Corollary 3.2.

Proof of Corollary 3.2 The subsolution property follows from Theorem 3.1 and Theorem 3.2 since $\check{g} \geq g$. As for the supersolution property, we note that Theorem 3.1 and Theorem 3.2 imply that, for fixed $t_{0} \in[0, T], v_{*}\left(t_{0}, \cdot\right)$ is a supersolution of (3.39). It thus follows from Lemma 3.6 that $v_{*} \geq \check{g}$. The supersolution property then follows. To conclude, we note that the comparison result of Proposition 3.1 obviously still holds if we replace $g$ by $\check{g}$ since $\check{g}$ is continuous with respect to its first variable by assumption, and with respect to its second one by Lemma 3.7.

Proof of Corollary 3.3. The fact that $\check{g}=\hat{g}$ follows from the discussion at the beginning of Section 3.4. Note that the continuity of $g$ implies that $\hat{g}$ is continuous too. Also observe that the fact that $\sigma$ is invertible implies that, for a smooth function $\varphi$,

$$
\sup _{\pi \in \mathcal{N} \varphi(t, x)}\left(\pi^{\prime} \mu(x) \varphi(t, x)-\mathcal{L} \varphi(t, x)\right)=-\partial_{t} \varphi-\frac{1}{2} \operatorname{Trace}\left[\operatorname{diag}[x] \sigma \sigma^{\prime} \operatorname{diag}[x] D^{2} \varphi\right] .
$$

Let us now observe that the map $w$ defined on $[0, T] \times(0, \infty)^{d}$ by

$$
w(t, x):=\sup _{\tau \in \mathcal{T}_{[t, T]}} \mathbb{E}^{\mathbb{Q}}\left[\hat{g}\left(\tau, X_{t, x}(\tau)\right)\right]
$$

is a viscosity solution on $[0, T) \times(0, \infty)^{d}$ of

$$
\min \left\{-\partial_{t} \varphi-\frac{1}{2} \operatorname{Trace}\left[\operatorname{diag}[x] \sigma \sigma^{\prime} \operatorname{diag}[x] D^{2} \varphi\right], \varphi-\hat{g}\right\}=0 .
$$

In particular, it is a subsolution of (3.12), recall (3.46). We next deduce from the definition of $\hat{g}$ that, for all $\rho \in \mathbb{R}^{d}$,

$$
w\left(t, x e^{\rho}\right)=\sup _{\tau \in \mathcal{T}_{[t, T]}} \mathbb{E}^{\mathbb{Q}}\left[\hat{g}\left(\tau, X_{t, x}(\tau) e^{\rho}\right)\right] \leq \sup _{\tau \in \mathcal{T}_{[t, T]}} \mathbb{E}^{\mathbb{Q}}\left[e^{\delta(\rho)} \hat{g}\left(\tau, X_{t, x}(\tau)\right)\right]=e^{\delta(\rho)} w(t, x) .
$$

It follows that $w(t, x) \geq e^{-\delta(\rho)} w\left(t, x e^{\rho}\right)$ for all $\rho \in \mathbb{R}^{d}$ which implies that $w$ is a viscosity supersolution of $\mathcal{M} \varphi=0$. Hence, $w$ is a supersolution of (3.12), recall (3.46). Finally, (3.40) and standard estimates show that $w$ satisfy the growth condition (3.11). It thus follows from Corollary 3.2 that $v_{*}=v^{*}=w$. 


\section{References}

[1] Barles G. (1994), Solutions de Viscosité des Equations d'Hamilton-Jacobi, Springer-Verlag, Berlin.

[2] Bensoussan A., N. Touzi and J.-L. Menaldi (2005), Penalty approximation and analytical characterization of the problem of super-replication under portfolio constraints, Asymptotic Analysis, 41, 311-330.

[3] Bertsekas D. P. and S. E. Shreve (1978), Stochastic Optimal Control: The Discrete Time Case, Mathematics in Science and Engineering, Academic Press, 139.

[4] Bouchard B. (2002), Stochastic Target with Mixed diffusion processes, Stochastic Processes and their Applications, 101, 273-302.

[5] Bouchard B. and I. Bentahar (2006), Barrier option hedging under constraints: a viscosity approach, SIAM J.Control Optim., 45 (5), 1846-1874.

[6] Bouchard B., R. Elie and C. Imber (2008), Optimal control under stochastic target constraints, forthcoming.

[7] Bouchard B., R. Elie and N. Touzi (2008), Stochastic target problems with controlled loss, preprint Ceremade.

[8] Broadie M., J. Cvitanic̀ and M. Soner (1998), Optimal replication of contingent claims under portfolio constraints. The Review of Financial Studies, 11 (1), 59-79.

[9] Crandall M. G., H. Ishii and P.-L Lions (1992), User's guide to viscosity solutions of second order partial differential equations, Amer. Math. Soc., 27, 1-67.

[10] Cvitanic̀ J. and I. Karatzas (1993), Hedging contingent claims with constrained portfolios, Annals of Applied Probability, 3, 652-681.

[11] Cvitanic̀ J. , H. Pham and N. Touzi (1999), Super-replication in stochastic volatility models with portfolio constraints, Journal of Applied Probability, 36, 523-545.

[12] Föllmer H. and D. Kramkov (1997), Optional decomposition under constraints, Probability Theory and Related Fields, 109, 1-25.

[13] Karatzas I. and S. G. Kou (1996), Hedging American contingent claims with constrained portfolios, Annals of Applied Probability, 6, 321-369.

[14] Karatzas I. and H. Wang (2000), A Barrier Option of American type, Applied Mathematics and Optimization, 42, 259-280.

[15] Karatzas I. and S. E. Shreve (1998), Methods of Mathematical Finance, Springer-Verlag, NewYork.

[16] El Karoui N. (1979), Les aspects probabilistes du contrôle stochastique, Ecole d'Eté de Probabilités de Saint Flour IX, Lecture Notes in Mathematics 876, Springer Verlag.

[17] El Karoui N. and M.-C. Quenez (1995), Dynamic Programming and Pricing of Contingent Claims in an Incomplete Market, SIAM J. on Control and Optimization, 33 (1), 22-66.

[18] Rockafellar R. T. (1970), Convex analysis, Princeton University Press, Princeton, NJ. 
[19] Shreve S. E., U. Schmock and U. Wystup (2002), Valuation of exotic options under shortselling constraints, Finance and Stochastics, 6, 143-172.

[20] Soner H. M. and N. Touzi (2000), Super-replication under Gamma constraint, SIAM J.Control Optim., 39, 73-96.

[21] Soner H. M. and N. Touzi (2002), Stochastic target problems, dynamic programming and viscosity solutions, SIAM J.Control Optim., 41, 2, 404-424.

[22] Soner H. M. and N. Touzi (2002), Dynamic programming for stochastic target problems and geometric flows, Journal of the European Mathematical Society, 4, 201-236.

[23] Soner H. M. and N. Touzi (2003), Stochastic representation of mean curvature type geometric flows, Annals of Probability, 31, 1145-1165.

[24] Soner H. M. and N. Touzi (2004), The problem of super-replication under constraints, ParisPrinceton Lectures in Mathematical Finance, Lecture Notes in Mathematics, Springer-Verlag. 